\title{
An Interior-Point algorithm for Nonlinear Minimax Problems
}

\author{
E. Obasanjo, $\quad$ G. Tzallas-Regas $\dagger \quad$ B. Rustem ${ }^{\ddagger}$
}

*Equity Derivatives Group - Systematic Trading, Barclays Capital, London, UK (Enitan.Obasanjo@barclayscapital.com)

${ }^{\dagger}$ Research Associate, Department of Computing, Imperial College London, London SW7 2AZ, UK (gt299@doc.ic.ac.uk)

${ }^{\ddagger}$ Professor, Department of Computing, Imperial College London, London SW7 2AZ, UK (br@doc.ic.ac.uk) 


\begin{abstract}
We present a primal-dual interior-point method for constrained nonlinear, discrete minimax problems where the objective functions and constraints are not necessarily convex. The algorithm uses two merit functions to ensure progress toward the points satisfying the first-order optimality conditions of the original problem. Convergence properties are described and numerical results provided.
\end{abstract}

Key Words. Discrete min-max, Constrained nonlinear programming, Primal-dual interior-point methods, Stepsize strategies. 


\section{Introduction}

A number of interior-point algorithms for linear and nonlinear programming have been developed in the years following the introduction of Karmarkar's method in Ref. 1. El-Bakry et al. provided the formulation and theory of the Newton interior-point method for nonlinear programming in Ref. 2, extending the general primal-dual framework proposed for LP problems by Kojima et al. (Ref. 3). Their algorithm used the $l_{2}$ norm of the residuals as merit function in the line-search procedure. This choice of merit function, however may lead the algorithm to converge to a saddle point or maximum when a minimum is being sought. Facilitated by advances in computing, the development of interior-point methods for nonlinear problems has been of great interest over the last two decades. In light of the success of some interior-point methods to this class of problems (see e.g. Ref. 4), the research presented in this paper aimed to develop an efficient and robust algorithm for general nonlinear minimax problems (Refs. 5, 6) using a primal-dual interiorpoint method. The resulting algorithm is presented in this paper.

The algorithm is based on the primal-dual interior-point method described in Ref. 7 and the minimax approach of Rustem (Ref. 8). It differs from Ref. 8 mainly in the choice of merit function, stepsize rule and computation of search direction. The objective and constraint functions of the optimization problem are assumed to be smooth and twice differentiable. Newton's method is used to solve the primal-dual system of the problem, generating descent directions for the merit function. The use of slack variables in the inequality constraints relaxes the requirement of feasibility at each iteration. An adaptive penalty parameter is employed to ensure progress towards optimality and feasibility. Two merit functions are used to ensure convergence towards a solution of the optimization problem.

The algorithm code is linked with the mathematical programming language AMPL, which provides first and second order derivative information, but the approach is also applicable to quasi-Newton methods. Global and local convergence results are established for the algorithm. The performance of the method is illustrated through some numerical examples, and is compared with the results obtained using MINOS, LOQO and IPOPT on the NEOS server 4.0 on problems drawn from the CUTE test set. 


\section{Description of Algorithm}

In this section we describe the algorithm we are going to present. First we discuss a reformulation of the minimax problem and then propose how to solve the reformulation with a primal-dual interior-point framework.

\subsection{Minimax Problem}

We consider the following equality constrained minimax problem:

$$
\min _{x \in R^{n}} \max _{j=1, \ldots, m} f^{j}(x) \text { subject to } g(x)=0, x \geq 0
$$

where $f^{j}: R^{n} \rightarrow R, j=1, \ldots, m$ and $g: R^{n} \rightarrow R^{q}$ are smooth, twice differentiable, nonlinear functions.

It has been shown in Ref. 8, from the original proof Medanić and Andjelić (Refs. 9, 10), that problem (1) is equivalent to the following min-max formulation with pooled objectives

$$
\min _{x \in R^{n}} \max _{\alpha \in \Xi_{+}^{m}}\{\langle\alpha, f(x)\rangle \mid g(x)=0, x \geq 0\},
$$

where

$$
\Xi_{+}^{m}=\left\{\alpha \in R^{m} \mid \alpha \geq 0,\langle\mathbf{e}, \alpha\rangle=1\right\},
$$

$\mathbf{e} \in R^{m}$ is a vector of all ones, and $f: R^{n} \rightarrow R^{m}$ with

$$
f(x)=\left[f_{1}(x), \ldots, f_{m}(x)\right]^{T} .
$$

This follows from the fact that the maximum of $m$ numbers is equal to the maximum of their convex combination.

By introducing an additional variable, $v$, the discrete minimax problem can be represented as the nonlinear program (NLP)

$$
\min _{x \in R^{n}, v \in R^{1}}\{v \mid f(x) \leq v \mathbf{e}, g(x)=0, x \geq 0\} .
$$

To enable comparison with other algorithms for nonlinear programming, the minimax problems discussed in this paper are implemented using the above NLP. However, both the equivalent formulations (2) and (4) are used in this paper to represent problem (1) whenever convenient. 


\subsection{Primal-Dual Method}

The use of slack variables, as was done in Ref. 11, yields the following reformulation of the minimax problem (4):

$$
\min _{x \in R^{n}, v \in R^{1}, s \in R^{m}}\{v \mid f(x)-v \mathbf{e}+s=0, g(x)=0, x \geq 0, s \geq 0\} .
$$

The equality constraints can be combined to form a new constraint vector $G: R^{n+m+1} \rightarrow R^{m+q}$ defined as

$$
G(v, x, s)=\left(\begin{array}{c}
f(x)+s-v \mathbf{e} \\
g(x)
\end{array}\right) .
$$

For notational compactness, the decision variables $x$ and slack variables $s$ can be combined into a single variable:

$$
\bar{x}=\left(\begin{array}{c}
x \\
s
\end{array}\right)
$$

where $\bar{x} \in R^{N}$ and $N=n+m$. Thus, we can re-write problem (5) as:

$$
\min _{\bar{x} \in R^{N}, v \in R^{1}}\{v \mid G(v, \bar{x})=0, \bar{x} \geq 0\} .
$$

The inequality constraints in (7) above can then be incorporated into a barrier term

$$
B(\bar{x})=\sum_{i=1}^{N} \log \bar{x}^{i}
$$

leading to the problem:

$$
\min _{\bar{x} \in R^{N}, v \in R^{1}}\{v-\mu B(\bar{x}) \mid G(v, \bar{x})=0\}
$$

for $\bar{x}>0$ with barrier parameter $\mu \geq 0$.

The Lagrangian function associated with the constrained optimization problem in (8) is given by

$$
L(v, \bar{x}, y ; \mu)=v+\langle y, G(v, \bar{x})\rangle-\mu B(\bar{x}),
$$

where $y \in R^{p}, p=q+m$, are the Lagrange multipliers of the equality constraints. 
The Karush-Kuhn-Tucker (KKT) optimality conditions for this problem are

$$
\begin{aligned}
& \nabla_{v} L(v, \bar{x}, y ; \mu)=1+\left\langle y, \nabla_{v} G(v, \bar{x})\right\rangle_{\bar{X}-1}=0, \\
& \nabla_{\bar{x}} L(v, \bar{x}, y ; \mu)=\nabla_{\bar{x}} G(v, \bar{x})^{T} y-\mu \bar{X}^{-1} \mathbf{e}=0, \\
& \nabla_{y} L(v, \bar{x}, y ; \mu)=G(v, \bar{x}) \quad=0,
\end{aligned}
$$

where $\bar{X}=\operatorname{diag}(\bar{x})$, is the diagonal matrix with elements $\bar{x}^{i}$, and $\nabla_{a} G(v, \bar{x})$ is the Jacobian matrix of $G(v, \bar{x})$ with respect to variable $a$.

By introducing the nonlinear transformation $z=\mu \bar{X}^{-1} \mathbf{e}$ we modify Eqs. (10) to produce the standard primal-dual system:

$$
E(v, \bar{x}, z, y ; \mu)=\left(\begin{array}{c}
1+\left\langle y, \nabla_{v} G(v, \bar{x})\right\rangle \\
\nabla_{\bar{x}} G(v, \bar{x})^{T} y-z \\
\bar{X} Z \mathbf{e}-\mu \mathbf{e} \\
G(v, \bar{x})
\end{array}\right)=0
$$

where $Z=\operatorname{diag}(z)$, with $(\bar{x}, z)>0$.

\subsection{Newton System and Search Direction}

Primal-dual methods use the Newton or quasi-Newton method to solve approximately the perturbed KKT conditions for a fixed value of $\mu$. The Newton system for (11) above is:

$$
\left[\begin{array}{cccc}
0 & 0 & 0 & -\mathbf{e}^{T} \\
0 & \nabla_{\bar{x} \bar{x}}^{2} L(v, \bar{x}, y ; \mu) & -I & \nabla_{\bar{x}} G(v, \bar{x})^{T} \\
0 & Z & \bar{X} & 0 \\
-\mathbf{e} & \nabla_{\bar{x}} G(v, \bar{x}) & 0 & 0
\end{array}\right]\left[\begin{array}{c}
\Delta v \\
\Delta \bar{x} \\
\Delta z \\
\Delta y
\end{array}\right]=-E(v, \bar{x}, z, y ; \mu),
$$

since

$$
\nabla_{v v}^{2} L(v, \bar{x}, y ; \mu)=0
$$

and

$$
\nabla_{v \bar{x}} L(v, \bar{x}, y ; \mu)=\nabla_{\bar{x} v} L(v, \bar{x}, y ; \mu)=0 .
$$

Let $w^{p}=(v, \bar{x})$ represent the primal variables and $w=(v, \bar{x}, z, y)$ all the variables of problem (7). System (12) is then solved to give the search directions $\Delta w=(\Delta v, \Delta \bar{x}, \Delta z, \Delta y)$. The algorithm proceeds iteratively from an initial point $\left(v_{0}, \bar{x}_{0}, z_{0}, y_{0}\right)$ through a sequence $w_{k+1}=w_{k}+\tau_{k} \Delta w_{k}$, where

$$
\tau_{k}=\operatorname{diag}\left(\tau_{p k} I_{v}, \tau_{p k} I_{\bar{x}}, \tau_{d k} I_{z}, \tau_{d k} I_{y}\right),
$$


with $\tau_{p k}$ being the primal stepsize, $\tau_{d k}$ the dual stepsize and $I_{a}$ being a unity matrix of dimension related to the length of $a$. System (12) can be expressed as:

$$
\nabla E(w ; \mu) \Delta w=-E(w ; \mu) .
$$

The Newton system (12) can be re-written as:

$$
\begin{aligned}
-\langle\mathbf{e}, \Delta y\rangle & =-[1-\langle y, \mathbf{e}\rangle] \\
Q \Delta \bar{x}+\nabla_{\bar{x}} G(v, \bar{x})^{T} \Delta y-\Delta z & =-\left[\nabla_{\bar{x}} G(v, \bar{x})^{T} y-z\right] \\
Z \Delta \bar{x}+\bar{X} \Delta z & =-(\bar{X} Z \mathbf{e}-\mu \mathbf{e}) \\
-\Delta v \mathbf{e}+\nabla_{\bar{x}} G(v, \bar{x}) \Delta \bar{x} & =-G(v, \bar{x})
\end{aligned}
$$

where $Q$ is the Hessian of the Lagrangian function, $L(v, \bar{x}, y ; \mu)$, or an approximation of the latter.

Remark 2.1 Due to Eqs. (13), (14) there holds

$$
\left\langle\Delta w^{p}, \nabla_{w^{p}}^{2} L(v, \bar{x}, y ; \mu) \Delta w^{p}\right\rangle=\left\langle\Delta \bar{x}, \nabla_{\bar{x}}^{2} L(v, \bar{x}, y ; \mu) \Delta \bar{x}\right\rangle=\langle\Delta \bar{x}, Q \Delta \bar{x}\rangle .
$$

Remark 2.2 From the definition of $w^{p}$, Eq. (16d) can be expressed as:

$$
\nabla_{w^{p}} G\left(w^{p}\right) \Delta w^{p}=-G\left(w^{p}\right),
$$

where

$$
\nabla_{w^{p}} G\left(w^{p}\right)=\left[\begin{array}{ll}
\nabla_{v} G(v, \bar{x}) & \nabla_{\bar{x}} G(v, \bar{x})
\end{array}\right],
$$

and $\Delta w^{p}=(\Delta v, \Delta \bar{x})$.

The design of the algorithm used to solve problem (8) is based on the following assumptions:

(A1) Smoothness: $G(v, \bar{x})$ is a smooth twice continuously differentiable function of $v$ and $\bar{x}$.

(A2) Regularity: The columns of the matrix $[\nabla G(v, \bar{x})]$ corresponding to the binding constraints are linearly independent.

(A3) Complementarity: At a solution point, $w_{*}=\left(v_{*}, \bar{x}_{*}, z_{*}, y_{*}\right)$, strict complementarity is satisfied, i.e. if $\bar{x}_{*}^{i}>0$ then $z_{*}^{i}=0$ and if $z_{*}^{i}>0, \bar{x}_{*}^{i}=0$ for $i=1,2, \ldots, N$. 
(A4) Second order sufficiency: The second order sufficiency condition is satisfied at a solution point.

(A5) Feasibility: The feasible region is bounded ${ }^{1}$.

(A6) There exists a constant $m_{1}>0$ such that

$$
\left\|Q_{k}\right\| \leq m_{1}, \quad \text { for all } k .
$$

(A7) There exists a constant $m_{2}>0$ such that

$$
m_{2}\|p\|^{2} \leq\left\langle p, Q_{k} p\right\rangle
$$

for all $k$ and all $0 \neq p \in R^{N}$ such that $\nabla_{\bar{x}} G\left(w_{k}^{p}\right) p=0$.

\subsection{Merit Functions}

In this section we describe the merit functions used for determining the stepsize of the algorithm.

Let $\epsilon_{G}>0$ be a finite precision to which the equality constraints are satisfied, i.e. we have a worst-case feasibility precision

$$
\left\|G\left(w_{k}^{p}\right)\right\|_{2}^{2}>\epsilon_{G} .
$$

While (17) holds, the inner iterations $k$ use the following merit function

$$
\Phi_{1}\left(w^{p} ; c, \mu\right)=v+(c / 2)\left\|G\left(w^{p}\right)\right\|_{2}^{2}-\mu B(\bar{x}) .
$$

For any inner iteration, $k$, where $0<\left\|G_{k}\right\|_{2}^{2} \leq \epsilon_{G}$, the algorithm switches to the second merit function,

$$
\Phi_{2}(w)=(1 / 2)\|E(w ; 0)\|_{2}^{2},
$$

for all consecutive inner iterations, where $E(w ; 0)$ is as defined in (11) with $\mu=0$. Once the convergence of the inner iterations is achieved, the algorithm switches back to the original merit function.

\footnotetext{
${ }^{1}$ This may be enforced, if required, in the usual way of placing upper and lower bounds on $\bar{x}$.
} 


\subsection{Penalty Parameter Selection Rule}

The penalty parameter selection strategy is an important part of the algorithm. At each iteration for which (17) holds, a value of $c$ is determined such that the descent property

$$
\Delta v_{k}-\mu \Delta \bar{x}_{k}^{T} \bar{X}_{k}^{-1} \mathbf{e}-c_{k}\left\|G\left(v_{k}, \bar{x}_{k}\right)\right\|_{2}^{2}+d_{k}\left(\Delta \bar{x}_{k}\right) \leq 0,
$$

associated with $\Phi_{1}$ holds. The monotonic descent of function $\Phi_{1}$ is discussed in Theorem 3.1. Inequality (20) plays an important role to the proof of Theorem 3.1. Term $d_{k}\left(\Delta \bar{x}_{k}\right)$ is a function of the search direction and should have the property that

$$
d_{k}\left(\Delta \bar{x}_{k}\right) \geq 0
$$

in order to prove Theorem 3.1 and generally establish global convergence of our algorithm. The exact choice of $d_{k}\left(\Delta \bar{x}_{k}\right)$ depends on the framework used. For example, in a quasi-Newton framework exploiting hereditary positivedefiniteness of Hessian updates the natural choice would be

$$
d_{k}\left(\Delta \bar{x}_{k}\right)=\left\langle\Delta \bar{x}_{k}, Q_{k} \Delta \bar{x}_{k}\right\rangle
$$

In such frameworks $Q_{k}$ is chosen positive semi-definite, therefore (21) is satisfied. In a Newton framework such a choice for $d_{k}\left(\Delta \bar{x}_{k}\right)$ is not entirely sufficient. In our algorithm we choose

$$
d_{k}\left(\Delta \bar{x}_{k}\right)=\left\{\begin{array}{ll}
\left\langle\Delta \bar{x}_{k}, Q_{k} \Delta \bar{x}_{k}\right\rangle, & \text { if } G_{k}=0 \\
\sigma_{k}\left\|\Delta \bar{x}_{k}\right\|^{2}, & \text { otherwise }
\end{array},\right.
$$

where $\sigma_{k} \in(0,1 / 2]$ can be constant throughout the algorithm, or can be updated from iteration to iteration ${ }^{1}$.

Remark 2.3 In order for $d_{k}\left(\Delta \bar{x}_{k}\right)$, as chosen by (23), to satisfy property (21), then when $G_{k}=0$, there must hold that $\left\langle\Delta \bar{x}_{k}, Q_{k} \Delta \bar{x}_{k}\right\rangle \geq 0$. If we take into account (16d), then $\left\langle\Delta \bar{x}_{k}, Q_{k} \Delta \bar{x}_{k}\right\rangle \geq 0$ if Assumption A7 is satisfied.

Since $\mu$ is fixed throughout the inner iterations, it can be deduced that the sign of the term on the left hand side of (20) is dependent on the value of c. For insufficiently large values of $c$, the descent property (20) may not be satisfied. When this is the case, the penalty parameter is updated to a new

\footnotetext{
${ }^{1}$ We do not present an iteration scheme for $\sigma_{k}$, and consider it a constant positive value.
} 
value $c_{k+1}>c_{k}$ which guarantees the satisfaction of (20). Lemma 3.2 shows that the algorithm chooses the value of the penalty parameter to satisfy (20). In Lemmas 3.2 and 3.4 we show that descent is always guaranteed if (17) holds or if $G\left(v_{k}, \bar{x}_{k}\right)=0$, and that the penalty parameter $c_{k}=c_{k}\left(\epsilon_{G}\right)$ remains finite, due to the use of two merit functions. This scheme is a variation of the watchdog technique, first suggested by Chamberlain et al. (Ref. 12), and used in the context of interior-point methods by Gay et al. (Ref. 13). The convergence property of an algorithm based on the merit function (19) is discussed in Ref. 2. The algorithm in Ref. 2 has two salient features that need to be noted. The first is the choice of penalty parameter selection rule for (18) which extends the sequential quadratic programming approach proposed in (Refs. 14, 15) for nonlinear programming and in Rustem and Nguyen (Ref. 16) for minimax. The second, and perhaps more important, aspect is the objective and method of application of the second merit function, defined in (19). We illustrate, through an example in Section 4, that the $l_{2}$ norm of the KKT conditions on its own is unsuitable for use as a merit function for non-convex optimization, but this is overcome with a combination of merit functions as defined in (18) and (19).

\subsection{Line Search Strategies}

The algorithm utilizes an Armijo line search strategy to determine stepsizes in the search direction. The search strategy should decrease the value of the merit function chosen by the algorithm.

When $\Phi_{1}$ is in use, then for , $\hat{\tau}>0, \beta \in(0,1)$ the stepsize strategy in the algorithm determines the stepsize $\tau_{p k}$ as the largest value of $\tau=\hat{\tau} \beta^{j}, j=$ $0,1,2, \ldots$ such that $w_{k+1}^{p}$ given by $\bar{x}_{k+1}=\bar{x}_{k}+\tau_{p k} \Delta \bar{x}_{k}, v_{k+1}=\max _{j} f^{j}\left(\bar{x}_{k+1}\right)$ satisfies the inequality

$$
\Phi_{1}\left(w_{k+1}^{p} ; c_{k+1}, \mu\right)-\Phi_{1}\left(w_{k}^{p} ; c_{k}, \mu\right) \leq \rho \tau_{p k} \phi_{1}\left(w_{k}^{p}, \Delta w_{k}^{p} ; c_{k+1}, \mu\right),
$$

where $\rho \in(0,1 / 2)$ is a given scalar and

$$
\phi_{1}\left(w^{p}, \Delta w^{p} ; c, \mu\right)=\left\langle\Delta w^{p}, \nabla_{w^{p}} \Phi_{1}\left(w^{p} ; c, \mu\right)\right\rangle
$$

is the directional derivative of $\Phi_{1}$ at $w_{k}^{p}$ along the primal search direction $\Delta w_{k}^{p}$. At iteration $k$, using the definition of $\Phi_{1}$ from (18) and Remark 2.2 we can write $\phi_{1}$ as

$$
\phi_{1}\left(w_{k}^{p}, \Delta w_{k}^{p} ; c_{k}, \mu\right)=\Delta v_{k}-\mu \Delta \bar{x}_{k}^{T} \bar{X}_{k}^{-1} \mathbf{e}-c_{k+1}\left\|G_{k}\right\|^{2} .
$$


When $\Phi_{2}$ is in use, then for $\hat{\tau}>0, \beta \in(0,1)$ the stepsize strategy in the algorithm determines the stepsize $\tau_{k}$ as the largest value of $\tau=\hat{\tau} \beta^{j}, j=$ $0,1, \ldots$, such that $w_{k+1}$ given by

$$
w_{k+1}=w_{k}+\tau_{k} \Delta w_{k}
$$

satisfies the inequality

$$
\Phi_{2}\left(w_{k}+\tau_{k} \Delta w_{k}\right)-\Phi_{2}\left(w_{k}\right) \leq \rho \tau_{k} \phi_{2}\left(w_{k}, \Delta w_{k}\right) .
$$

where, again, $\phi_{2}$ is the directional derivative of $\Phi_{2}$ at $w_{k}$ along the search direction $\Delta w_{k}$. At iteration $k$ we can write $\phi_{2}$, using (15), as

$$
\phi_{2}\left(w_{k}, \Delta w_{k}\right)=\left\langle\Delta w_{k}, \nabla_{w} \Phi_{2}\left(w_{k}\right)\right\rangle=\left\langle\nabla E^{T}\left(w_{k} ; 0\right) E\left(w_{k} ; 0\right), \Delta w_{k}\right\rangle .
$$

Algorithm 1 : Primal-Dual Interior-Point algorithm

Step 0: Initialization:

Given $\tilde{\bar{x}}^{0} \in R^{N}, \tilde{\bar{x}}^{0}>0$.

Choose $\tilde{y}^{0} \in R^{p}, \tilde{z}^{0} \in R^{N}, \tilde{z}^{0}>0$.

Choose penalty and barrier parameters $c_{0}>0, \mu^{0}>0$.

Choose $\beta, \epsilon_{0}, \epsilon_{G}, \eta, \theta \in(0,1), \rho \in(0,1 / 2), \delta, M_{0}, \omega>0, \sigma_{k}=1$.

Set $\tilde{v}^{0}=\max _{j} f^{j}\left(\tilde{\bar{x}}^{0}\right)$.

Set $l=0, k=0$ and new-merit $=$ false.

Step 1: Convergence of outer iterations:

If $\left\|E\left(\tilde{v}^{l}, \tilde{\bar{x}}^{l}, \tilde{z}^{l}, \tilde{y}^{l} ; \mu^{l}\right)\right\|_{2} /\left(1+\left\|\left(\tilde{v}^{l}, \tilde{\bar{x}}^{l}, \tilde{z}^{l}, \tilde{y}^{l}\right)\right\|_{2}\right) \leq \epsilon_{0}$ then STOP.

Step 2: Inner iterations: ( $\mu$ is fixed to $\mu^{l}$ throughout this step)

Set $\left(v_{k}, \bar{x}_{k}, z_{k}, y_{k}\right)=\left(\tilde{v}^{l}, \tilde{\bar{x}}^{l}, \tilde{z}^{l}, \tilde{y}^{l}\right)$

Step 2.1: Convergence of inner iterations:

If $\| E\left(v_{k}, \bar{x}_{k}, z_{k}, y_{k} ; \mu^{l} \|_{2} \leq \eta \mu^{l}\right.$ and $\left\|G_{k}\right\|_{2}^{2} \leq \epsilon_{G}$ then set $\left(\tilde{v}^{l+1}, \tilde{\bar{x}}^{l+1}, \tilde{z}^{l+1}, \tilde{y}^{l+1}\right)=\left(v_{k}, \bar{x}_{k}, z_{k}, y_{k}\right)$ and go to Step 3.

Step 2.2: Search direction generation:

Solve Newton system (12) to obtain $\Delta w_{k}=\left(\Delta v_{k}, \Delta \bar{x}_{k}, \Delta z_{k}, \Delta y_{k}\right)$.

Step 2.3: Penalty parameter selection:

Compute $d_{k}\left(\Delta \bar{x}_{k}\right)$ using (23). 
Set $c_{k+1}=c_{k}$.

If new-merit is false then set

$$
\mathcal{M}_{\text {num }}=\Delta v_{k}-\mu \Delta \bar{x}_{k}^{T} \bar{X}_{k}^{-1} \mathbf{e}-c_{k}\left\|G_{k}\right\|^{2}+d_{k}\left(\Delta \bar{x}_{k}\right)
$$

else go to Step 2.4

If new-merit is false and $\mathcal{M}_{\text {num }}>0$ and $\left\|G_{k}\right\|_{2}^{2}>\epsilon_{G}$ then set

$$
c_{k+1}=\max \left\{\left(\Delta v_{k}-\mu \Delta \bar{x}_{k}^{T} \bar{X}_{k}^{-1} \mathbf{e}+d_{k}\left(\Delta \bar{x}_{k}\right)\right) /\left\|G_{k}\right\|^{2}, c_{k}+\delta\right\}
$$

Step 2.4: Step-length selection:

Set $\tau_{p k}^{\max }=\min _{1 \leq i \leq N}\left\{-\bar{x}_{k}^{i} / \Delta \bar{x}_{k}^{i} \mid \Delta \bar{x}_{k}^{i}<0\right\}$.

If $\left(\mathcal{M}_{\text {num }}>0\right.$ and $\left.0<\left\|G_{k}\right\|_{2}^{2} \leq \epsilon_{G}\right)$ or new-merit is true then

Set $\tau_{d k}^{\max }=\min _{1 \leq i \leq N}\left\{-z_{k}^{i} / \Delta z_{k}^{i} \mid \Delta z_{k}^{i}<0\right\}$.

Set $\hat{\tau}_{k}=\min \left\{1, \theta \tau_{p k}^{\max }, \theta \tau_{d k}^{\max }\right\}$.

Let $\tau_{k}=\beta^{j} \hat{\tau}_{k}$, where $j$ is the smallest non-negative integer:

$$
\Phi_{2}\left(w_{k}+\tau_{k} \Delta w_{k}\right)-\Phi_{2}\left(w_{k}\right) \leq \rho \tau_{k} \phi_{2}\left(w_{k}, \Delta w_{k}\right) .
$$

Set new-merit $=$ true.

else

Set $\hat{\tau}_{p k}=\min \left\{1, \theta \tau_{p k}^{\max }\right\}$.

Let $\tau_{p k}=\beta^{j} \hat{\tau}_{p k}$, where $j$ is the smallest non-negative integer:

$\Phi_{1}\left(w_{k+1}^{p} ; c_{k+1}, \mu\right)-\Phi_{1}\left(w_{k}^{p} ; c_{k+1}, \mu\right) \leq \rho \tau_{p k} \phi_{1}\left(w_{k}^{p}, \Delta w_{k}^{p} ; c_{k+1}, \mu\right)$

with $\bar{x}_{k+1}=\bar{x}_{k}+\tau_{p k} \Delta \bar{x}_{k}$ and $v_{k+1}=\max _{j} f^{j}\left(x_{k+1}\right)$.

Choose $\hat{m}, \hat{M}>0$.

Set

$$
\begin{aligned}
L B_{k}^{i} & =\min \left\{(1 / 2) \hat{m} \mu, \bar{x}_{k+1}^{i} z_{k}^{i}\right\} \\
U B_{k}^{i} & =\min \left\{2 \hat{M} \mu, \bar{x}_{k+1}^{i} z_{k}^{i}\right\}
\end{aligned}
$$

Set for $i=1, \ldots N$

$$
\tau_{d k}^{i}=\max \left\{\tau_{i}>0: L B_{k}^{i} \leq \bar{x}_{k+1}^{i}\left(z_{k}^{i}+\tau_{i} \Delta z_{k}^{i}\right) \leq U B_{k}^{i}\right\}
$$




$$
\text { Set } \tau_{d k}=\min \left\{1, \min _{1 \leq i \leq N}\left\{\tau_{d k}^{i}\right\}\right\}
$$

Set

$$
y_{k+1}=y_{k}+\tau_{d k} \Delta y_{k}, \quad z_{k+1}=z_{k}+\tau_{d k} \Delta z_{k} .
$$

Step 2.5: Start a new inner iteration:

Set $k=k+1$ and go to Step 2.1.

Step 3: Barrier parameter adjustment:

Reduce the barrier parameter $\mu$ as described in Section 2.8.

Step 4: Start a new outer iteration:

Set new-merit $=$ false

Set $l=l+1$ and go to Step 1 .

\subsection{Stepsize Rules}

In this section we discuss the step size rules associated with $\Phi_{1}$. A treatment of the step size rules associated with $\Phi_{2}$ can be found in Ref. 2 .

Primal Stepsize Rule. For the primal variables, $(x, s)$, represented by $\bar{x}$, we determine the maximum allowable stepsizes by the boundary of the feasible region:

$$
\tau_{p k}^{\max }=\min _{1 \leq i \leq N}\left\{-\bar{x}_{k}^{i} / \Delta \bar{x}_{k}^{i} \mid \Delta \bar{x}_{k}^{i}<0\right\} .
$$

This is indeed the maximum step allowed because $\tau_{p k}^{\max }$ gives an infinitely large value to at least one term of the logarithmic barrier function $B\left(\bar{x}_{k+1}\right)$. However, if the stepsize is in the interval $\left[0, \tau_{p k}^{\max }\right)$ then the next primal iterate $\bar{x}_{k+1}$ is strictly feasible and none of the logarithmic terms become infinitely large. As an initial step, $\hat{\tau}_{p k}$, we choose a number close to $\tau_{p k}^{\max }$ but never greater than one, i.e. $\hat{\tau}_{p k}=\min \left[\theta \tau_{p k}^{\max }, 1\right]$. with $\theta \in(0,1)$. The Armijo rule is then applied to give $\tau_{p k}=\beta^{j} \hat{\tau}_{p k}$, with $\beta$ chosen to be in the interval $[0.1,0.5]$, dependent on the confidence on the initial step $\hat{\tau}_{p k}$.

Dual Stepsize Rule. The dual stepsize strategy used by the algorithm follows that described in Ref. 7. It is a modification of the strategy suggested by Yamashita (Ref. 17) and Yamashita and Yabe (Ref. 18). 
For a fixed value of the barrier parameter $\mu$, we determine a stepsize $\tau_{d k}^{i}$ along the direction $\Delta z_{k}^{i}$ for each dual variable $z_{k}^{i}, i=1,2, \ldots, N$, such that the following box constraints are satisfied:

$$
\tau_{d k}^{i}=\max \left\{\tau_{i}>0: L B_{k}^{i} \leq \bar{x}_{k+1}^{i}\left(z_{k}^{i}+\tau_{i} \Delta z_{k}^{i}\right) \leq U B_{k}^{i}\right\}
$$

with $L B_{k}^{i}$ and $U B_{k}^{i}$ defined, as in Step 2.4 of the algorithm, as

$$
\begin{aligned}
L B_{k}^{i} & =\min \left\{(1 / 2) \hat{m} \mu, \bar{x}_{k+1}^{i} z_{k}^{i}\right\} \\
U B_{k}^{i} & =\min \left\{2 \hat{M} \mu,\left(\bar{x}_{k+1}^{i}\right)\left(z_{k}^{i}\right)\right\} .
\end{aligned}
$$

The parameters $\hat{m}$ and $\hat{M}$, as proposed in Ref. 19, are chosen to satisfy

$$
0<\hat{m} \leq \min \left\{1,\left((1-\eta)\left(1-\eta / M_{0}^{\mu^{l}}\right) \min _{i}\left\{\left(\tilde{x}^{l}\right)^{i}\left(\tilde{z}^{l}\right)^{i}\right\}\right) / \mu^{l}\right\}
$$

and

$$
\hat{M} \geq \max \left\{1, \max _{i}\left\{\left(\tilde{\bar{x}}^{l}\right)^{i}\left(\tilde{z}^{l}\right)^{i}\right\} / \mu^{l}\right\}>0,
$$

for a fixed value of $\mu^{l}$, with $\eta \in(0,1)$ and $M_{0}$ a large positive number.

The common dual step $\tau_{d k}$, is the minimum of all individual step-lengths $\tau_{d k}^{i}$ with the restriction of always being no greater than one. Numerically:

$$
\tau_{d k}=\min \left\{1, \min _{1 \leq i \leq N}\left\{\tau_{d k}^{i}\right\}\right\} .
$$

The step-lengths for the dual variables $y$ can be set to either $\tau_{d k}$ or 1 . It is clear from the construction of the lower and upper bounds that

$$
L B_{k}^{i} /\left(\bar{x}_{k}^{i}+\tau_{p k} \Delta \bar{x}_{k}^{i}\right) \leq z_{k}^{i} \leq U B_{k}^{i} /\left(\bar{x}_{k}^{i}+\tau_{p k} \Delta \bar{x}_{k}^{i}\right)
$$

for all $i=1, \ldots, N$.

The dual stepsize rule defines a neighborhood of the central path beyond which the iterates cannot stray. It does so by setting a lower bound which prevents the products $\bar{x}_{k}^{i} z_{k}^{i}$ from becoming very much smaller than the current value of the barrier parameter, preventing them reaching the boundary of the feasible region prematurely. The dual stepsize rule also prevents the variables $\bar{x}_{k}^{i}$ and $z_{k}^{i}$ from becoming unbounded by setting an upper bound on them that is proportional to the barrier parameter. 


\subsection{Selection of Barrier Parameter}

The selection of the barrier parameter follows the strategy outlined in Ref. 7. It is restated here for convenience

$$
\begin{aligned}
& \text { Set } \mu^{l+1}=\min \left\{0.95 \mu^{l}, 0.01(0.95)^{k}\left\|E\left(w_{k} ; 0\right)\right\|_{2}\right\} \\
& \text { if }\left\|E\left(w_{k} ; \mu^{l}\right)\right\|_{2} \leq 0.1 \eta \mu^{l} \text { and } \mu^{l}<10^{-4} \text { the set } \\
& \qquad \mu^{l+1}=\min \left\{0.95 \mu^{l}, 0.01(0.95)^{k+2 \omega}\left\|E\left(w_{k} ; 0\right)\right\|_{2}\right\} . \\
& \text { if }\left\|E\left(w_{k} ; \mu^{l}\right)\right\|_{2} \leq 0.1 \eta \mu^{l} \text { and } \mu^{l} \geq 10^{-4} \text { the set } \\
& \qquad \mu^{l+1}=\min \left\{0.95 \mu^{l}, 0.01(0.95)^{k+\omega}\left\|E\left(w_{k} ; 0\right)\right\|_{2}\right\} .
\end{aligned}
$$

In the above, $\omega>0$ is a parameter which accelerates the decrease of $\mu$ at appropriate points. The algorithm checks if the current point is close enough to the central path (i.e. if $\left.\left\|E\left(w_{k} ; \mu^{l}\right)\right\|_{2} \leq 0.1 \eta \mu^{l}\right)$ and to the optimum solution (i.e if $\mu^{l}<10^{-4}$ ), in which case the barrier parameter is multiplied by $(0.95)^{2 \omega}$, reducing it at a faster rate. If the current point is close to the central path but not an optimum solution, the barrier parameter is multiplied by the larger factor $(0.95)^{\omega}$.

\section{Convergence Results}

In this section the algorithm is shown to be globally convergent, in the sense that it guarantees progress towards a solution of the approximate KKT conditions (11) from any starting point and for a fixed value of the barrier parameter $\mu^{l}=\mu$.

Lemma 3.1 Let $f$ and $g$ be differentiable functions and let there exist a small $\epsilon_{G}>0$ such that $\left\|G_{k}\right\|_{2}^{2}>\epsilon_{G}$. If $\Delta \bar{x}_{k}$ is the search direction for variable $\bar{x}$ calculated by the solution of the Newton system (12), $c_{k+1}$ is chosen as in

Step 2.4 of the algorithm above and $d_{k}(\Delta \bar{x})$ satisfies (21), then $\Delta \bar{x}_{k}$ is a descent direction for $\Phi_{1}$. Furthermore

$$
\phi_{1}\left(w_{k}^{p}, \Delta w_{k}^{p} ; c_{k+1}, \mu\right) \leq-d_{k}\left(\Delta \bar{x}_{k}\right) \leq 0 .
$$


Proof. Step 2.3 of the algorithm checks if

$$
\Delta v_{k}-\mu \Delta \bar{x}_{k}^{T} \bar{X}_{k}^{-1} \mathbf{e}-c_{k}\left\|G_{k}\right\|^{2}+d_{k}\left(\Delta \bar{x}_{k}\right) \leq 0 .
$$

If this is satisfied then by setting $c_{k+1}=c_{k}$ and rearranging (32) we obtain

$$
\phi_{1}\left(w_{k}^{p}, \Delta w_{k}^{p} ; c_{k+1}, \mu\right) \leq-d_{k}\left(\Delta \bar{x}_{k}\right) \leq 0 .
$$

On the other hand, if (32) is not satisfied, then by setting

$$
c_{k+1} \geq\left(\Delta v_{k}-\mu \Delta \bar{x}_{k}^{T} \bar{X}_{k}^{-1} \mathbf{e}+d_{k}\left(\Delta \bar{x}_{k}\right)\right) /\left\|G_{k}\right\|^{2}
$$

and substituting into (26), it can be verified from (21) that

$$
\phi_{1}\left(w_{k}^{p}, \Delta w_{k}^{p} ; c_{k+1}, \mu\right) \leq-d_{k}\left(\Delta \bar{x}_{k}\right) \leq 0 .
$$

This proves the lemma.

The above lemma establishes the descent property of the search direction for $\left\|G_{k}\right\|_{2}^{2}>\epsilon_{G}$. We now proceed to demonstrate the descent property for the merit function when $\left\|G_{k}\right\|_{2}^{2}=0$. We stress that assumption A7 is needed in order to prove descent for $\Phi_{1}$ in this lemma only.

Lemma 3.2 Let $f$ and $g$ be differentiable functions and also let $A 7$ hold. If $\left(\Delta v_{k}, \Delta \bar{x}_{k}, \Delta y_{k}, \Delta z_{k}\right)$ is the solution to the Newton system (12) and $G_{k}=0$ for some $k$, then the descent property

$$
\phi_{1}\left(w_{k}^{p}, \Delta w_{k}^{p} ; c_{k}, \mu\right) \leq-d_{k}\left(\Delta \bar{x}_{k}\right) \leq 0
$$

is satisfied for any choice of the penalty parameter, $c_{k} \in[0, \infty)$. Therefore the search direction $\Delta \bar{x}_{k}$ obtained from the solution of the Newton system of equations is still a descent direction after feasibility of the equality constraints has been achieved.

Proof. If $G_{k}=0,(26)$ can be written as:

$$
\phi_{1}\left(w_{k}^{p}, \Delta w_{k}^{p} ; c_{k+1}, \mu\right)=\Delta v_{k}-\mu \Delta \bar{x}_{k}^{T} \bar{X}_{k}^{-1} \mathbf{e} .
$$

We can also write (16d) as:

$$
-\Delta v_{k} \mathbf{e}+\nabla_{\bar{x}} G_{k} \Delta \bar{x}_{k}=0 .
$$


Multiplying the last one by $\left(y_{k}+\Delta y_{k}\right)^{T}$ and using (16a) we get

$$
\Delta v_{k}=\left\langle\Delta \bar{x}_{k}, \nabla_{\bar{x}} G_{k}^{T}\left(y_{k}+\Delta y_{k}\right)\right\rangle .
$$

Rearranging (16b) we get

$$
\nabla_{\bar{x}} G_{k}^{T}\left(y_{k}+\Delta y_{k}\right)=-Q_{k} \Delta \bar{x}_{k}+z_{k}+\Delta z_{k}
$$

and if we multiply it on the left by $\Delta \bar{x}_{k}^{T}$ we obtain

$$
\left\langle\Delta \bar{x}_{k}, \nabla_{\bar{x}} G_{k}^{T}\left(y_{k}+\Delta y_{k}\right)\right\rangle=-\left\langle\Delta \bar{x}_{k}, Q_{k} \Delta \bar{x}_{k}\right\rangle+\left\langle\Delta \bar{x}_{k}, z_{k}+\Delta z_{k}\right\rangle .
$$

and therefore write (35) as

$$
\Delta v_{k}=-\left\langle\Delta \bar{x}_{k}, Q_{k} \Delta \bar{x}_{k}\right\rangle+\left\langle\Delta \bar{x}_{k}, z_{k}+\Delta z_{k}\right\rangle .
$$

Next, solving equation (16c) for $\Delta z_{k}+z_{k}$ gives

$$
\Delta z_{k}+z_{k}=-\bar{X}_{k}^{-1} Z_{k} \Delta \bar{x}_{k}+\mu \bar{X}_{k}^{-1} \mathbf{e}
$$

and multiplying it by $\Delta \bar{x}_{k}^{T}$ yields

$$
\left\langle\Delta \bar{x}_{k}, \Delta z_{k}+z_{k}\right\rangle=-\Delta \bar{x}_{k}^{T} \bar{X}_{k}^{-1} Z_{k} \Delta \bar{x}_{k}+\mu \Delta \bar{x}_{k}^{T} \bar{X}_{k}^{-1} \mathbf{e} .
$$

Then we substitute $\left\langle\Delta \bar{x}_{k}, \Delta z_{k}+z_{k}\right\rangle$ from (37) in (36) to obtain

$$
\Delta v_{k}=-\left\langle\Delta \bar{x}_{k}, Q_{k} \Delta \bar{x}_{k}\right\rangle-\Delta \bar{x}_{k}^{T} \bar{X}_{k}^{-1} Z_{k} \Delta \bar{x}_{k}+\mu \Delta \bar{x}_{k}^{T} \bar{X}_{k}^{-1} \mathbf{e}
$$

Finally, using (38) in (34) we can write

$$
\begin{aligned}
\phi_{1}\left(w_{k}^{p}, \Delta w_{k}^{p} ; c_{k+1}, \mu\right)= & -\left\langle\Delta \bar{x}_{k}, Q_{k} \Delta \bar{x}_{k}\right\rangle-\Delta \bar{x}_{k}^{T} \bar{X}_{k}^{-1} Z_{k} \Delta \bar{x}_{k} \\
& +\mu \Delta \bar{x}_{k}^{T} \bar{X}_{k}^{-1} \mathbf{e}-\mu \Delta \bar{x}_{k}^{T} \bar{X}_{k}^{-1} \mathbf{e} \\
= & -\left\langle\Delta \bar{x}_{k}, Q_{k} \Delta \bar{x}_{k}\right\rangle-\Delta \bar{x}_{k}^{T} \bar{X}_{k}^{-1} Z_{k} \Delta \bar{x}_{k} \\
\leq & -\left\langle\Delta \bar{x}_{k}, Q_{k} \Delta \bar{x}_{k}\right\rangle \\
= & -d_{k}\left(\Delta \bar{x}_{k}\right) \leq 0 .
\end{aligned}
$$

The last inequality holds since $x, z$ are kept strictly feasible, and the last equality from the definition of $d_{k}\left(\Delta \bar{x}_{k}\right)$ in (23).

Lemma 3.3 Let $f$ and $g$ be differentiable. If $\Delta w_{k}$ is the direction calculated by solving the Newton system (12) with $c_{k+1}$ chosen as in Algorithm 1, then $\Delta w_{k}$ is a descent direction for $\Phi_{2}$ when $0<\left\|G_{k}\right\|_{2}^{2} \leq \epsilon_{G}$. 
Proof. When $0<\left\|G_{k}\right\|_{2}^{2} \leq \epsilon_{G}$, Step 2.4 of the algorithm uses $\Phi_{2}\left(w_{k}\right)$. Using (15) we can write

$$
\left\langle\Delta w_{k}, \nabla \Phi_{2}\left(w_{k}\right)\right\rangle=\left\langle\Delta w_{k},\left(\nabla E_{k}^{T} E_{k}\right)\right\rangle=-\left\|E_{k}\right\|^{2} \leq 0
$$

from which the descent property is established.

Corollary 3.1 If $\left\|\Delta w_{k}\right\|=0$, the algorithm chooses $c_{k+1}=c_{k}$.

Proof. From (16d) and the definition of $G_{k}$ when $\Delta w_{k}=0, G_{k}=0$, therefore this situation is covered by Lemma 3.2 (See Ref. 7).

Lemma 3.4 Let $f$ and $g$ satisfy Assumption A1. Then, for a fixed value $\mu^{l}$ of $\mu$ :

(i) There exists a constant $c_{k+1} \geq 0$, satisfying Step 2.3 of the algorithm.

(ii) Assuming that the sequence $\bar{x}_{k}$ is bounded, $c_{k}$ is increased finitely often. That is, there exists an integer $k_{*} \geq 0$, and $c_{*} \geq 0$, such that for all $k \geq k_{*}, c_{k}=c_{*}<\infty$.

Proof. Part (i) follows directly from the preceding Lemmas, since a finite value $c_{k+1}$ is always generated in Step 2.3. Part (ii) can be shown by contradiction. Assume that $c_{k} \rightarrow \infty$ as $k \rightarrow \infty$. From the definition of $c_{k+1}$ in Step 2.3, we can deduce that if $c_{k} \rightarrow \infty$ then $\left\|G_{k}\right\|_{2}^{2} \rightarrow 0$. Hence, there exists an integer $k_{1}$ such that for all $k \geq k_{1}$ we have $\left\|G_{k}\right\|_{2}^{2} \leq \epsilon_{G}$. In Step 2.4 of the

algorithm, however, when $\left\|G_{k}\right\|_{2}^{2} \leq \epsilon_{G}$, the algorithm stops increasing the penalty parameter and switches to the second merit function. Therefore, the maximum value that $c_{k}$ can attain is $c_{*}=c_{k_{1}}=M_{\text {num }} / \epsilon_{G}$ where $M_{\text {num }}$ and $c_{*}$ are finite values. Hence, $c_{*}<\infty$, contrary to our assumption that $c_{k} \rightarrow \infty$ as $k \rightarrow \infty$. Hence, the penalty parameter does not increase indefinitely and there exists an integer $k_{*} \geq 0$ such that for all $k \geq k_{*}$ we have $c_{k}<\infty$.

\subsection{Convergence of the Inner Iterations}

Consider the objective function

$$
\max _{\alpha \in \Xi_{m}^{+}} L(x, \alpha)=\max _{\alpha \in \Xi_{m}^{+}}\langle\alpha, f\rangle=\max _{j}\left(f^{j}\right),
$$


and its linear approximation with respect to $x$ at $x_{k}$

$$
\begin{aligned}
\max _{\alpha \in \Xi_{m}^{+}} L_{k}(x, \alpha) & =\max _{\alpha \in \Xi_{m}^{+}}\left\langle\alpha, f\left(x_{k}\right)+\nabla f^{T}\left(x_{k}\right)\left(x-x_{k}\right)\right\rangle \\
& =\max _{j}\left(f_{k}^{j}+\left\langle\nabla f_{k}^{j}, \Delta x_{k}\right\rangle\right) .
\end{aligned}
$$

This objective function is used in the following theorem.

Theorem 3.1 Monotonic Descent of $\Phi_{1}$

- Let Assumptions A1, A6, AY hold,

- For each $k$, let there exist a bounded point $\Delta w_{k}=\left(\Delta v_{k}, \Delta \bar{x}_{k}, \Delta z_{k}, \Delta y_{k}\right)$ as a solution to the Newton system (12),

- Let there exist an iteration $k_{*}$, small $\epsilon_{G}>0,\left\|G_{k}\right\|_{2}^{2} \notin\left(0, \epsilon_{G}\right)$ and a scalar $c_{*} \geq 0, c_{*}=c_{*}\left(\epsilon_{G}\right)$, such that the condition

$$
\Delta v_{k}-\mu \Delta \bar{x}_{k}^{T} \bar{X}_{k}^{-1} \mathbf{e}-c_{k}\left(\epsilon_{G}\right)\left\|G_{k}\right\|^{2}+d_{k}\left(\Delta \bar{x}_{k}\right) \leq 0,
$$

is satisfied for all $k \geq k_{*}$ and $c_{k+1}\left(\epsilon_{G}\right)=c_{k}\left(\epsilon_{G}\right)=c_{*}\left(\epsilon_{G}\right)$.

Then the stepsize computed in Step 2.4 is such that $\tau_{p k} \in(0,1]$ and hence the sequence $\left\{\Phi_{1}\left(w_{k}^{p} ; c_{k}, \mu\right)\right\}$ is monotonically decreasing for $k \geq k_{*}$ and $\mu$ fixed.

Proof. From Step 2.4 of the algorithm we wish to find a bound for

$$
\Delta \Phi_{1}=\Phi_{1}\left(w_{k+1}^{p} ; c_{k+1}, \mu\right)-\Phi_{1}\left(w_{k}^{p} ; c_{k}, \mu\right)
$$

for $\bar{x}_{k+1}=\bar{x}_{k}+\tau_{p k} \Delta \bar{x}_{k}, v_{k+1}=\max _{j}\left\{f^{j}\left(x_{k+1}\right)\right\}^{2}$.

We have that

$$
\begin{aligned}
u_{k+1} & =\max _{\alpha \in \Xi_{m}^{+}}\left\{\left\langle\alpha, f\left(x_{k}\right)+\tau_{p k} \Delta x_{k}^{T} \nabla f\left(x_{k}\right)\right\rangle\right\} \\
& =\max _{\alpha \in \Xi_{m}^{+}}\left\{L_{k}\left(x_{k}+\tau_{p k} \Delta x_{k}, \alpha\right)\right\}
\end{aligned}
$$

where $L_{k}$ is defined in (39). From the convexity of $\max _{\alpha \in \Xi_{m}^{+}}\left\{L_{k}(x, \alpha)\right\}$

$$
\begin{aligned}
u_{k+1} & \leq \max _{\alpha \in \Xi_{m}^{+}}\left\{L_{k}\left(x_{k}, \alpha\right)\right\}+\tau_{p k}\left(\max _{\alpha \in \Xi_{m}^{+}}\left\{L_{k}\left(x_{k}+\tau_{p k} \Delta x_{k}, \alpha\right)\right\}-\max _{\alpha \in \Xi_{m}^{+}}\left\{L_{k}\left(x_{k}, \alpha\right)\right\}\right) \\
& \leq u_{k}+\tau_{p k}\left(\left(u_{k}+\Delta u_{k}\right)-v_{k}\right) \\
& =u_{k}+\tau_{p k} \Delta v_{k} .
\end{aligned}
$$

\footnotetext{
${ }^{2}$ The dependency of $\Delta \Phi_{1}$ on $\left(w_{k+1}^{p}, w_{k}^{p}, c_{k+1}, c_{k}, \mu\right)$ has been dropped so as to avoid long equations.
} 
Using the definition of $\Phi_{1}$ from (18) we can write (40) as

$$
\begin{aligned}
\Delta \Phi_{1} & =u_{k+1}-u_{k}+\left(c_{*} / 2\right)\left(\left\|G_{k+1}\right\|^{2}-\left\|G_{k}\right\|^{2}\right)+\mu\left(B\left(\bar{x}_{k+1}\right)-B\left(\bar{x}_{k}\right)\right) \\
& \leq \tau_{p k} \Delta v_{k}-\tau_{p k} c_{*}\left\|G_{k}\right\|^{2}-\tau_{p k} \mu\left\langle\Delta \bar{x}_{k}, \bar{X}_{k}^{-1} \mathbf{e}\right\rangle+\left(\tau_{p k}^{2} / 2\right) F_{\Phi_{1}} \\
& =\tau_{p k}\left(\Delta v_{k}-c_{*}\left\|G_{k}\right\|^{2}-\mu\left\langle\Delta \bar{x}_{k}, \bar{X}_{k}^{-1} \mathbf{e}\right\rangle\right)+\left(\tau_{p k}^{2} / 2\right) F_{\Phi_{1}} \\
& =\tau_{p k} \phi_{1}\left(\Delta w_{k}^{p}, w_{k}^{p} ; c_{*}, \mu\right)+\left(\tau_{p k}^{2} / 2\right) F_{\Phi_{1}} .
\end{aligned}
$$

The first term on the right of the inequality comes from (41); the second and third terms come from a Taylor series expansion of $G(x)$ and $B(\bar{x})$, respectively; we use the fact that $c_{k}=c_{k+1}=c_{*}$ for $k \geq k_{*}$ according to Lemma 3.4; second order terms of the Taylor expansion are in $F_{\Phi_{1}}$, which is defined as

$$
F_{\Phi_{1}}=\int_{0}^{1}(1-t)\left\langle\Delta \bar{x}_{k}, \nabla_{\bar{x}_{k}^{2}}^{2} \Phi_{1}\left(v_{k}, \bar{x}_{k}+t \tau_{p k} \Delta \bar{x}_{k} ; c_{*}, \mu\right) \Delta \bar{x}_{k}\right\rangle d t .
$$

Finally, the last equality comes from from (26).

If we add and subtract $\left(\tau_{p k}^{2} / 2\right)\left\langle\Delta \bar{x}_{k}, Q_{k} \Delta \bar{x}_{k}\right\rangle$ from the right hand side, we can write

$$
\begin{aligned}
\Delta \Phi_{1} \leq & \tau_{p k} \phi_{1}\left(\Delta w_{k}^{p}, w_{k}^{p} ; c_{*}, \mu\right)+\left(\tau_{p k}^{2} / 2\right) F_{\Phi_{1}} \\
& +\left(\tau_{p k}^{2} / 2\right)\left(\left\langle\Delta \bar{x}_{k}, Q_{k} \Delta \bar{x}_{k}\right\rangle-\left\langle\Delta \bar{x}_{k}, Q_{k} \Delta \bar{x}_{k}\right\rangle\right) \\
= & \tau_{p k} \phi_{1}\left(\Delta w_{k}^{p}, w_{k}^{p} ; c_{*}, \mu\right)+\left(\tau_{p k}^{2} / 2\right)\left\langle\Delta \bar{x}_{k}, Q_{k} \Delta \bar{x}_{k}\right\rangle \\
& +\left(\tau_{p k}^{2} / 2\right)\left(F_{\Phi_{1}}-\left\langle\Delta \bar{x}_{k}, Q_{k} \Delta \bar{x}_{k}\right\rangle\right) \\
\leq & \tau_{p k} \phi_{1}\left(\Delta w_{k}^{p}, w_{k}^{p} ; c_{*}, \mu\right)+\left(\tau_{p k}^{2} / 2\right)\left\langle\Delta \bar{x}_{k}, Q_{k} \Delta \bar{x}_{k}\right\rangle \\
& +\left(\tau_{p k}^{2} / 2\right) \xi_{k}\left\|\Delta \bar{x}_{k}\right\|^{2}
\end{aligned}
$$

where

$$
\xi_{k}=\int_{0}^{1}(1-t)\left\|\nabla_{\bar{x}_{k}^{2}}^{2} \Phi_{1}\left(v_{k}, \bar{x}_{k}+t \tau_{p k} \Delta \bar{x}_{k} ; c_{*}, \mu\right)-Q_{k}\right\| d t .
$$

Using the Cauchy-Schwarz inequality and Assumption A6 we can write

$$
\left\langle\Delta \bar{x}_{k}, Q_{k} \Delta \bar{x}_{k}\right\rangle \leq\left\|Q_{k}\right\|\left\|\Delta \bar{x}_{k}\right\|^{2} \leq m_{1}\left\|\Delta \bar{x}_{k}\right\|^{2}
$$

therefore inequality (42) can be written as

$$
\begin{aligned}
\Delta \Phi_{1} & \leq \tau_{p k} \phi_{1}\left(\Delta w_{k}^{p}, w_{k}^{p} ; c_{*}, \mu\right)+\left(\tau_{p k}^{2} / 2\right) m_{1}\left\|\Delta \bar{x}_{k}\right\|^{2}+\left(\tau_{p k}^{2} / 2\right) \xi_{k}\left\|\Delta \bar{x}_{k}\right\|^{2} \\
& =\tau_{p k} \phi_{1}\left(\Delta w_{k}^{p}, w_{k}^{p} ; c_{*}, \mu\right)+\left(\tau_{p k}^{2} / 2\right)\left(m_{1}+\xi_{k}\right)\left\|\Delta \bar{x}_{k}\right\|^{2} .
\end{aligned}
$$


In Lemmas 3.1, 3.2 we have shown that for $\left\|G_{k}\right\|^{2} \notin\left(0, \epsilon_{G}\right)$

$$
\phi_{1}\left(\Delta w_{k}^{p}, w_{k}^{p} ; c_{*}, \mu\right) \leq-d_{k}\left(\Delta \bar{x}_{k}\right) .
$$

If we choose $M_{1}=\min \left\{\sigma_{k}, m_{2}\right\}$, then from (23)

$$
d_{k}\left(\Delta \bar{x}_{k}\right) \geq M_{1}\left\|\Delta \bar{x}_{k}\right\|^{2}
$$

therefore combining the last two

$$
\left\|\Delta \bar{x}_{k}\right\|^{2} \leq-\left(1 / M_{1}\right) \phi_{1}\left(\Delta w_{k}^{p}, w_{k}^{p} ; c_{*}, \mu\right) .
$$

In this case we can write

$$
\begin{aligned}
\Delta \Phi_{1} & \leq \tau_{p k} \phi_{1}\left(\Delta w_{k}^{p}, w_{k}^{p} ; c_{*}, \mu\right)-\left(\tau_{p k}^{2} / 2 M_{1}\right)\left(m_{1}+\xi_{k}\right) \phi_{1}\left(\Delta w_{k}^{p}, w_{k}^{p} ; c_{*}, \mu\right) \\
& =\tau_{p k} \phi_{1}\left(\Delta w_{k}^{p}, w_{k}^{p} ; c_{*}, \mu\right)\left(1-\tau_{p k}\left(\left(m_{1}+\xi_{k}\right) / 2 M_{1}\right)\right) .
\end{aligned}
$$

The stepsize strategy in Step 2.4 of the algorithm determines $\tau_{p k}$ such that

$$
\rho \leq 1-\tau_{p k}\left(\left(m_{1}+\xi_{k}\right) / 2 M_{1}\right) \leq 1 / 2
$$

Since by Lemmas 3.2-3.4 the descent property $\phi_{1}\left(w_{k}^{p}, \Delta w_{k}^{p} ; c_{k+1}, \mu\right) \leq 0$ holds, there exists a $\tau_{p k}$ to ensure that (44) and hence Eq. (24) holds. Therefore $\left\{\Phi_{1}\left(w_{k}^{p} ; c_{k}, \mu\right)\right\}$ is monotonically decreasing.

Corollary 3.2 The sequence $\left\{\bar{x}_{k}\right\}$ generated by the algorithm is bounded away from zero.

Proof. Assume, to the contrary, that the sequence $\left\{\left\|\bar{x}_{k}\right\|\right\} \rightarrow 0$. Then

$$
\left\{B\left(\bar{x}_{k}\right)\right\} \rightarrow \infty
$$

From Assumption A5 (the feasible region is bounded), the sequences $f\left(x_{k}\right)$, $\left\{\left\|G\left(v_{k}, \bar{x}_{k}\right)\right\|\right\}$ and $\left\{\bar{x}_{k}\right\}$ must be bounded. Hence the sequence

$$
\left\{\Phi_{1}\left(w_{k}^{p} ; c_{*}, \mu\right)\right\} \rightarrow \infty
$$

This contradicts the monotonic decrease of this sequence established in Theorem 3.1. 
Lemma 3.5 For fixed $\mu$, the lower bounds $L B_{k}^{i}$ and the upper bounds $U B_{k}^{i}$, $i=1,2 \ldots n$ of the box constraints in the dual stepsize rule are bounded away from zero and bounded from above respectively, if the corresponding $\bar{x}_{k}^{i}$, of the iterates $\bar{x}_{k}$ are also bounded above and away from zero.

Proof. Established in Ref. 18 for the dual stepsize rule.

This also implies that $\left\{z_{k}\right\}$ is a sequence bounded above and away from zero. We have thus established that both $\left\{\bar{x}_{k}\right\}$ and $\left\{z_{k}\right\}$ are bounded sequences. Next we attempt to show that $\left\{y_{k}\right\}$ is also bounded. We establish an intermediate result in the next lemma and then proceed to prove that a unit step along $\Delta y_{k}$ results in sequence $\left\{y_{k}+\Delta y_{k}\right\}$ being bounded.

In the next paragraph we derive a reduced Newton system for our algorithm. Iterate subscripts and function arguments are omitted.

From the Newton system (16), solving (16c) for $\Delta z_{k}$ we obtain

$$
\Delta z=-\bar{X}^{-1} Z \Delta \bar{x}-z+\mu \bar{X}^{-1} \mathbf{e} .
$$

Substituting the latter in (16b), we can write (16) as

$$
\begin{aligned}
-\langle\mathbf{e}, \Delta y\rangle & =-[1-\langle y, \mathbf{e}\rangle] \\
Q \Delta \bar{x}+\nabla_{\bar{x}} G(v, \bar{x})^{T} \Delta y+\bar{X}^{-1} Z \Delta \bar{x}+z+\mu \bar{X}^{-1} \mathbf{e} & =-\nabla_{\bar{x}} G(v, \bar{x})^{T} y+z \\
-\Delta v \mathbf{e}+\nabla_{\bar{x}} G(v, \bar{x}) \Delta \bar{x} & =-G(v, \bar{x})
\end{aligned}
$$

which after cancellations and some re-arrangement gives

$$
\begin{aligned}
& -\langle\mathbf{e}, \Delta y+y\rangle \quad=-1 \\
& \Delta v \mathbf{e} \quad-\quad \nabla_{\bar{x}} G(v, \bar{x}) \Delta \bar{x}=G(v, \bar{x}) \\
& \nabla_{\bar{x}} G(v, \bar{x})^{T}(\Delta y+y)+\left(Q+\bar{X}^{-1} Z\right) \Delta \bar{x}=-\mu \bar{X}^{-1} \mathbf{e}
\end{aligned}
$$

which in matrix form is written as

$$
\left(\begin{array}{ccc}
0 & -\mathbf{e}^{T} & 0 \\
\mathbf{e} & 0 & -\nabla_{\bar{x}} G(v, \bar{x}) \\
0 & \nabla_{\bar{x}} G(v, \bar{x})^{T} & Q+\bar{X}^{-1} Z
\end{array}\right)\left(\begin{array}{c}
\Delta v \\
y+\Delta y \\
\Delta \bar{x}
\end{array}\right)=-\left(\begin{array}{c}
1 \\
-G(v, \bar{x}) \\
\mu \bar{X}^{-1} \mathbf{e}
\end{array}\right) .
$$

Lemma 3.6 Let $w_{k}$ be a sequence of vectors generated by our algorithm. Then the matrix sequence $\left\{R_{k}^{-1}\right\}$ is bounded, where

$$
R_{k}=\left(\begin{array}{ccc}
0 & -\mathbf{e}^{T} & 0 \\
\mathbf{e} & 0 & -\nabla_{\bar{x}} G(v, \bar{x}) \\
0 & \nabla_{\bar{x}} G(v, \bar{x})^{T} & Q+\bar{X}^{-1} Z
\end{array}\right)
$$


Proof. For simplicity, we shall suppress arguments and subscripts. The reduced matrix can be written as

$$
R=\left(\begin{array}{cc}
0 & A \\
-A^{T} & B
\end{array}\right)=\left(\begin{array}{ccc}
0 & -\mathbf{e}^{T} & 0 \\
\mathbf{e} & 0 & -\nabla_{\bar{x}} G \\
0 & \nabla_{\bar{x}} G^{T} & Q+\bar{X}^{-1} Z
\end{array}\right)
$$

where

$$
A=\left(\begin{array}{ll}
-\mathbf{e} & 0
\end{array}\right), \quad B=\left(\begin{array}{cc}
0 & -\nabla_{\bar{x}} G \\
\nabla_{\bar{x}} G^{T} & Q+\bar{X}^{-1} Z
\end{array}\right) .
$$

By assumptions A1, A2, Corollary 3.2 and Lemma 3.5 matrix $B^{-1}$ exists and is bounded, with

$$
B^{-1}=\left(\begin{array}{cc}
{\left[\nabla_{\bar{x}} G \Omega \nabla_{\bar{x}} G^{T}\right]^{-1}} & -\left[\nabla_{\bar{x}} G \Omega \nabla_{\bar{x}} G^{T}\right]^{-1} \nabla_{\bar{x}} G \Omega \\
\Omega \nabla_{\bar{x}} G^{T}\left[\nabla_{\bar{x}} G \Omega \nabla_{\bar{x}} G^{T}\right]^{-1} & \Omega-\Omega \nabla_{\bar{x}} G^{T}\left[\nabla_{\bar{x}} G \Omega \nabla_{\bar{x}} G^{T}\right]^{-1} \nabla_{\bar{x}} G \Omega
\end{array}\right)
$$

where $\Omega=\left(Q+\bar{X}^{-1} Z\right)^{-1}$. In addition $R_{k}$ is invertible with

$$
R^{-1}=\left(\begin{array}{cc}
{\left[A B^{-1} A^{T}\right]^{-1}} & -\left[A B^{-1} A^{T}\right]^{-1} A B^{-1} \\
B^{-1} A^{T}\left[A B^{-1} A^{T}\right]^{-1} & B^{-1}-B^{-1} A^{T}\left[A B^{-1} A^{T}\right]^{-1} A B^{-1}
\end{array}\right)
$$

and is bounded, since all the matrices involved are bounded.

Lemma 3.7 Let $\left\{w_{k}\right\}$ be a sequence of vectors generated by the algorithm. Then the sequence of vectors, $\left\{\Delta v_{k}, \Delta \bar{x}_{k}, y_{k}+\Delta y_{k}, \Delta z_{k}\right\}$ is bounded.

Proof. We have shown in Lemma 3.6 that the matrix on the left of the reduced system (45b) exists and is bounded. Therefore the sequences $\left\{\Delta v_{k}\right\}$, $\left\{\Delta \bar{x}_{k}\right\}$ and $\left\{y_{k}+\Delta y_{k}\right\}$ are also bounded. From (45a) and since all elements on the left are bounded, we deduce that $\left\{\Delta z_{k}\right\}$ is also bounded.

Lemma 3.8 Let the assumptions of Theorem 3.1 be satisfied and let, for some $k_{0}$ and $k \geq k_{0}$, the set

$$
U=\left\{w^{p} \in R^{N+1} \mid \Phi_{1}\left(w^{p} ; c_{*}, \mu\right) \leq \Phi_{1}\left(w_{k_{0}}^{p} ; c_{*}, \mu\right)\right\}
$$

be compact. Then for all $k \geq k_{0}$

$$
\lim _{k \rightarrow \infty} \phi_{1}\left(w_{k}^{p}, \Delta w_{k}^{p} ; c_{*}, \mu\right)=0 .
$$


Proof. The scalar $\rho \in(0,1 / 2)$ determined by the stepsize strategy of Step 2.4 is such that

$$
\rho \leq 1-\tau_{p k}\left(\left(m_{1}+\xi_{k}\right) / 2 M_{1}\right) \leq 1 / 2 .
$$

By solving for $\tau_{p k}$ we can write,

$$
\tau_{p k} \leq 2 M_{1}\left((1-\rho) /\left(m_{1}+\xi_{k}\right)\right)
$$

From the last one, the largest value $\tau_{p k}$ can take whilst ensuring satisfaction of the Armijo rule in Step 2.4 is

$$
\tilde{\tau}_{p k}=\min \left\{1,2 M_{1}\left((1-\rho) /\left(m_{1}+\xi_{k}\right)\right)\right\} .
$$

According to Step 2.4, $\tau_{p k}$ is chosen by reducing by a factor of $\beta$ the maximum allowed step length $\hat{\tau}_{p k}$ until inequality (24) is satisfied. Therefore $\tau_{p k} \in$ $\left[\beta \tilde{\tau}_{p k}, \tilde{\tau}_{p k}\right]$ and such a choice also satisfies $(24)$.

As $f$ and $g$ are twice continuously differentiable and $U$ is a compact set, there is a scalar $\bar{\xi}<\infty$ such that $\xi_{k} \leq \bar{\xi}$. Thus, since $M_{1}, m_{1}>0$, we have

$$
\tau_{p k} \geq \bar{\tau}_{p k}>0
$$

for all $k$, for the stepsize strategy defined by inequality (24) and for some

$$
\bar{\tau}_{p k}=\min \left\{1,2 M_{1}\left((1-\rho) /\left(m_{1}+\bar{\xi}\right)\right)\right\} .
$$

It is then obvious that the stepsize $\tau_{p k}$ is always bounded away from zero.

In addition, from Armijo's rule and Lemmas 3.1, 3.2 we have

$$
\Phi_{1}\left(w_{k+1}^{p} ; c_{*}, \mu\right)-\Phi_{1}\left(w_{k}^{p} ; c_{*}, \mu\right) \leq \rho \tau_{p k} \phi_{1}\left(w_{k}^{p}, \Delta w_{k}^{p} ; c_{*}, \mu\right)<0 .
$$

Having assumed that the level set $U$ is bounded, we can deduce that

$$
\lim _{k \rightarrow \infty}\left|\Phi_{1}\left(w_{k+1}^{p} ; c_{*}, \mu\right)-\Phi_{1}\left(w_{k}^{p} ; c_{*}, \mu\right)\right|=0
$$

which when combined with (48) and the fact that $\rho, \tau_{p k}>0$ proves the lemma.

Lemma 3.9 Let the assumptions of Lemma 3.8 be satisfied. Then

$$
\lim _{k \rightarrow \infty}\left\|\Delta \bar{x}_{k}\right\|=0
$$

and

$$
\lim _{k \rightarrow \infty}\left\|\Delta v_{k}\right\|=0 .
$$

Equivalently

$$
\lim _{k \rightarrow \infty}\left\|\Delta w_{k}^{p}\right\|=0
$$


Proof. We have shown in (43) that for $\left\|G_{k}\right\|^{2} \notin\left(0, \epsilon_{G}\right)$

$$
\left\|\Delta \bar{x}_{k}\right\|^{2} \leq-\left(1 / M_{1}\right) \phi_{1}\left(\Delta w_{k}^{p}, w_{k}^{p} ; c_{*}, \mu\right)
$$

for $M_{1}=\min \left\{\sigma_{k}, m_{2}\right\}$. Since $M_{1}>0$, (49a) holds from the previous lemma. Bearing in mind Remark 2.1 we derive the validity of $(49 \mathrm{c})$ which in turn yields the validity of $(49 \mathrm{~b})$.

Theorem 3.2 Let the assumptions of the previous lemma hold. Then for $\mu$ fixed, the algorithm asymptotically generates a point that satisfies the perturbed KKT conditions (11).

Proof. Let $\left(v_{*}(\mu), \bar{x}_{*}(\mu), z_{*}(\mu), y_{*}(\mu)\right)$ be a point such that $\left\{v_{k}\right\} \rightarrow v_{*}(\mu)$, $\left\{\bar{x}_{k}\right\} \rightarrow x_{*}(\mu),\left\{z_{k}\right\} \rightarrow z_{*}(\mu), k_{*} \leq k \in K$. The existence of such a point is ensured since - by Assumption A2, Corollary 3.2 and Lemmas 3.5 and 3.7the sequence of points $\left(v_{*}(\mu), \bar{x}_{*}(\mu), z_{*}(\mu), y_{*}(\mu)\right)$ is bounded for $\mu$ fixed and by Theorem 3.1, the algorithm sufficiently decreases $\Phi_{1}$, ensuring thus that $\left(v_{k}, \bar{x}_{k}\right) \in U$, with $U$ compact.

We begin our proof by showing that for $k$ sufficiently large, $\tau_{d k}$ becomes unity by establishing that

$$
\lim _{k \rightarrow \infty}\left\|z_{k}+\Delta z_{k}-\mu \bar{X}_{k+1}^{-1} \mathbf{e}\right\|=0
$$

Adding $-\mu X_{k+1}^{-1} \mathbf{e}$ to both sides of equation (45a) used in the reduced system and rearranging gives

$$
\left\|z_{k}+\Delta z_{k}-\mu \bar{X}_{k+1}^{-1} \mathbf{e}\right\| \leq\left\|-\bar{X}_{k}^{-1} Z_{k}\right\|\left\|\Delta \bar{X}_{k}\right\|+\mu\left\|\bar{X}_{k}^{-1}-\bar{X}_{k+1}^{-1}\right\|\|\mathbf{e}\| .
$$

Furthermore,

$$
\begin{aligned}
\left\|\bar{X}_{k}^{-1}-\bar{X}_{k+1}^{-1}\right\|_{2}^{2} & \leq N \max _{1 \leq i \leq N}\left\{\left(\left(1 / \bar{x}_{k}^{i}\right)-\left(1 / \bar{x}_{k+1}^{i}\right)\right)^{2}\right\} \\
& =N \max _{1 \leq i \leq N}\left\{\left(\tau_{p k}\right)^{2}\left(\Delta x_{k}^{i}\right)^{2} /\left(\bar{x}_{k}^{i}\right)^{2}\left(\bar{x}_{k+1}^{i}\right)^{2}\right\} .
\end{aligned}
$$

Since $\tau_{p k} \in(0,1],\left(\Delta \bar{x}_{k}^{i}\right)^{2} \leq\left\|\Delta \bar{x}_{k}\right\|_{2}^{2}$ and the sequence $\left\{\bar{x}_{k}\right\}$ is bounded away from zero, from the above inequality and (49a), we can derive

$$
\lim _{k \rightarrow \infty}\left\|\bar{X}_{k}^{-1}-\bar{X}_{k+1}^{-1}\right\|_{2}^{2} \leq N \lim _{k \rightarrow \infty} \max _{1 \leq i \leq N}\left\{\left\|\Delta \bar{x}_{k}\right\|_{2}^{2} /\left(\bar{x}_{k}^{i}\right)^{2}\left(\bar{x}_{k+1}^{i}\right)^{2}\right\}=0
$$


Consequently, letting $k \rightarrow \infty$ in (51) and using (49a) and (52), we can deduce that

$$
\lim _{k \rightarrow \infty}\left\|z_{k}+\Delta z_{k}-\mu \bar{X}_{k+1}^{-1} \mathbf{e}\right\|=0
$$

As a result, for sufficiently large $k$,

$$
z_{k+1}=z_{k}+\Delta z_{k}
$$

Moreover, using $\Delta z_{k}=-z_{k}+\mu \bar{X}_{k}^{-1} \mathbf{e}-\bar{X}_{k}^{-1} Z_{k} \Delta \bar{x}_{k}$, and for $k$ sufficiently large, the complementarity condition becomes

$$
\bar{X}_{k+1} z_{k+1}=\bar{X}_{k+1}\left(z_{k}+\Delta z_{k}\right)=\bar{X}_{k+1} \bar{X}_{k}^{-1}\left(\mu \mathbf{e}-Z_{k} \Delta \bar{x}_{k}\right)
$$

From (49a) and the fact that the elements of the diagonal matrix $\bar{X}_{k+1} \bar{X}_{k}^{-1}$ can be written as $\left(\bar{x}_{k+1}^{i}\right) /\left(\bar{x}_{k}^{i}\right)=1+\tau_{p k} \Delta \bar{x}_{k}^{i} /\left(\bar{x}_{k}^{i}\right)$, for all $i=1,2, \ldots, m$, it is clear that

$$
\lim _{k \rightarrow \infty} \bar{X}_{k+1} \bar{X}_{k}^{-1}=I_{N}
$$

where $I_{N}$ is the $N \times N$ identity matrix. Therefore, letting $k \rightarrow \infty$ in (54) and using (49a) and (55) yields

$$
\lim _{k \rightarrow \infty} \bar{X}_{k+1} z_{k+1}=\bar{X}_{*}(\mu) z_{*}(\mu)=\mu \mathbf{e} .
$$

Also, for $k \rightarrow \infty$, Eqs (16d) and (49) yield:

$$
\lim _{k \rightarrow \infty} \nabla G_{k} \Delta \bar{x}_{k}=G\left(\bar{x}_{*}(\mu)\right)=0 .
$$

For $z_{k+1}=z_{k}+\Delta z_{k}, y_{k+1}=y_{k}+\Delta y_{k}, \mathrm{Eq}(16 \mathrm{~b})$ can be written as

$$
\nabla G_{k}^{T} y_{k+1}-z_{k+1}=-Q_{k} \Delta \bar{x}_{k}
$$

Letting $k \rightarrow \infty$ in the above and using (49a), we get

$$
\lim _{k \rightarrow \infty}\left\|\nabla G_{k}^{T} y_{k+1}-z_{k+1}\right\|=0
$$

or equivalently

$$
\nabla G\left(\bar{x}_{*}(\mu)\right)^{T} y_{*}(\mu)-z_{*}(\mu)=0 .
$$

Also, from (53) and considering equation (16a),

$$
1=\left\langle y_{k+1}, \mathbf{e}\right\rangle
$$


and therefore letting $k \rightarrow \infty$, we get

$$
1=\left\langle y_{*}(\mu), \mathbf{e}\right\rangle \text {. }
$$

From (62), (60), (58) and (57), we conclude that $\left(v_{*}(\mu), \bar{x}_{*}(\mu), z_{*}(\mu), y_{*}(\mu)\right)$ is a solution of the perturbed KKT conditions given in (12).

A consequence of Theorem 3.2 is that, for any convergent subsequence produced by the algorithm, for $\mu=\mu^{l}$, there is an iteration $k \geq \tilde{k}$, such that

$$
\left\|E\left(\tilde{v}_{k}, \bar{x}_{\tilde{k}}, z_{\tilde{k}}, y_{\tilde{k}} ; \mu\right)\right\|_{2} \leq \eta \mu^{l}
$$

for all $k \geq \tilde{k}$ where $\eta \geq 0$. Record the value of the current iterate as

$$
\left(\tilde{v}^{l}, \tilde{\bar{x}}^{l}, \tilde{z}^{l}, \tilde{y}^{l}\right)=\left(\tilde{v}_{k}, \bar{x}_{\tilde{k}}, z_{\tilde{k}}, y_{\tilde{k}}\right)
$$

and set $\mu^{l+1}<\mu^{l}$. In this way we generate a sequence of approximate central points $\left\{\tilde{v}^{l}, \tilde{\bar{x}}^{l}, \tilde{z}^{l}, \tilde{y}^{l}\right\}$.

\subsection{Convergence of the Outer Iterations}

In this section it will be shown that the sequence of approximate central points converge to a KKT point $w_{*}=\left(v_{*}, \bar{x}_{*}, z_{*}, y_{*}\right)$ of the original constrained minimax problem (7).

For a given and sufficiently small $\varepsilon \geq 0$, considering the set of all approximate central points, generated by Algorithm 1,

$$
S(\varepsilon)=\left\{\tilde{w}^{l}: \varepsilon \leq\left\|E\left(\tilde{w}^{l} ; \mu^{l}\right)\right\|_{2} \leq\left\|E\left(\tilde{w}^{0} ; \mu^{0}\right)\right\|_{2}, \forall \mu^{l}<\mu^{0}\right\} .
$$

If $\varepsilon>0$, the stepsize rules described in previous sections guarantee that $\tilde{x}^{l}$ and $\tilde{z}^{l} \in S(\varepsilon)$ are bounded away from zero for $l \geq 0$. As a result $\left(\tilde{\bar{x}}^{l}\right)^{T}\left(\tilde{z}^{l}\right)$ are also bounded away from zero in $S(\varepsilon)$. The following lemma shows that the sequence $\left\{\tilde{y}^{l}\right\}$ is bounded if the sequence $\left\{\tilde{z}^{l}\right\}$ is also bounded.

Lemma 3.10 If Assumption A2 holds, and the iterates $\tilde{x}^{l}$ are in a compact set for $l \geq 0$, then there exists a constant $M_{1}>0$ such that

$$
\left\|\tilde{y}^{l}\right\| \leq M_{1}\left(1+\left\|\tilde{z}^{l}\right\|\right)
$$


Proof. We define $\tilde{r}^{l}=\nabla G\left(\tilde{x}^{l}\right)^{T} \tilde{y}^{l}-\tilde{z}^{l}$, and solve for $\nabla G\left(\tilde{x}^{l}\right) \tilde{y}^{l}$ to obtain

$$
\nabla G\left(\tilde{x}^{l}\right)^{T} \tilde{y}^{l}=\tilde{z}^{l}+\tilde{r}^{l} .
$$

From the assumptions made, the above equation can be written as:

$$
\tilde{y}^{l}=\left[\nabla G\left(\tilde{x}^{l}\right) \nabla G\left(\tilde{x}^{l}\right)^{T}\right]^{-1} \nabla G\left(\tilde{x}^{l}\right)\left(\tilde{z}^{l}+\tilde{r}^{l}\right) .
$$

Taking norms of both sides of the above equation yields:

$$
\begin{aligned}
\left\|\tilde{y}^{l}\right\| & \leq\left\|\left[\nabla G\left(\tilde{x}^{l}\right) \nabla G\left(\tilde{x}^{l}\right)^{T}\right]^{-1} \nabla G\left(\tilde{x}^{l}\right)\right\|\left(\left\|\left(\tilde{z}^{l}\right)\right\|+\left\|\tilde{r}^{l}\right\|\right) \\
& \leq M_{1}\left(1+\left\|\tilde{z}^{l}\right\|\right)
\end{aligned}
$$

where the constant $M_{1}$ is defined as

$$
M_{1} \geq\left[\nabla G\left(\tilde{x}^{l}\right) \nabla G\left(\tilde{x}^{l}\right)^{T}\right]^{-1} \nabla G\left(\tilde{x}^{l}\right) \max \left\{1,\left\|\tilde{r}^{l}\right\|\right\} .
$$

and is finite, according to our assumptions.

Lemma 3.11 If the sequence $\left\{\tilde{v}^{l}, \tilde{x}^{l}, \tilde{z}^{l}, \tilde{y}^{l}\right\}$ stays in $S(\varepsilon)$ for all $l \geq 0$, then it is bounded above.

Proof. From Lemma 3.10 it suffices to show that the sequences $\left\{\tilde{v}^{l}\right\},\left\{\tilde{\bar{x}}^{l}\right\}$, and $\left\{\tilde{z}^{l}\right\}$ are bounded from above. Sequence $\left\{\tilde{x}^{l}\right\}$ is bounded from above; since $f$ is continuous, by A5 we have that $\left\{\tilde{v}^{l}\right\}$ is also bounded. Therefore we only need to show that $\left\{\tilde{z}^{l}\right\}$ is bounded from above. This proof can be found in Ref. 7.

Theorem 3.3 Let $\left\{\mu^{l}\right\}$ be a positive monotonically decreasing sequence of barrier parameters with $\left\{\mu^{l}\right\} \rightarrow 0$, and let $\left\{\tilde{v}^{l}, \tilde{\bar{x}}^{l}, \tilde{z}^{l}, \tilde{y}^{l}\right\}$ be a sequence of approximate central points satisfying (63) for $\mu=\mu^{l}, l \geq 0$. Then, the sequence $\left\{\tilde{v}^{l}, \tilde{\bar{x}}^{l}, \tilde{z}^{l}, \tilde{y}^{l}\right\}$ is bounded and all of its limit points $\left(\tilde{v}^{*}, \tilde{\bar{x}}^{*}, \tilde{z}^{*}, \tilde{y}^{*}\right)$ satisfy the KKT conditions of problem (8).

Proof. From Lemma 3.11 the sequence $\left\{\tilde{v}^{l}, \tilde{\bar{x}}^{l}, \tilde{z}^{l}, \tilde{y}^{l}\right\}$ is bounded and remains in the compact set $S(\varepsilon)$. Thus it has a limit point in $S(\varepsilon)$, denoted by $\left(\tilde{v}^{*}, \tilde{\tilde{x}}^{*}, \tilde{z}^{*}, \tilde{y}^{*}\right)$. From (63) and the fact that $\left\{\mu^{l}\right\} \rightarrow 0$, we easily obtain that

$$
\lim _{l \rightarrow \infty}\left\|E\left(\tilde{v}^{l}, \tilde{\bar{x}}^{l}, \tilde{z}^{l}, \tilde{y}^{l} ; \mu\right)\right\|_{2}=0
$$

and $\left(\tilde{v}^{*}, \tilde{\bar{x}}^{*}, \tilde{z}^{*}, \tilde{y}^{*}\right)$ is a KKT point of the constrained problem (8). 


\section{Numerical Results}

In this section we present numerical experience related to the concepts presented. We first start by giving two examples that illustrate the reason why $\Phi_{2}$ on its own is not suitable for use as a merit function for non-convex optimization. The examples also highlight the usefulness of the proposed method to overcome the problem. Subsequently we present some numerical results obtained using an implementation of our method on the CUTE test set. Finally we describe the progress of our algorithm on two of the test problems, to note that the switch between the two merit functions indeed occurs.

Example 4.1 Consider the following problem

$$
\begin{aligned}
\min & f \\
\text { s.t } & -x^{3} \leq f, \\
& x^{3}+1 \leq f \\
& x+1 \leq f .
\end{aligned}
$$

As seen in Figure 1 it has a saddle point at $x=-0.19$ and a minimum at $x=-0.7937$. Suppose that we start at $x_{0}=(1.2)$.

1. Using solely $\Phi_{2}$, after 7 iterations we converge to $f^{*}=0.99$, which is the above the saddle point.

2. Using $\Phi_{1}$, and $\Phi_{2}$ as in Algorithm 1, after 9 iterations we converge to $f^{*}=0.5$ which is the above sought minimum.

Example 4.2 Consider the following example

$$
\begin{array}{cl}
\min & L \\
\text { s.t. } & x y-1+x^{2}+(y-1)^{2} \leq L, \\
& x y+1-x^{2}-(y-1)^{2} \leq L
\end{array}
$$

As seen in Figure 2 it has a maximum at $(x, y)=(0.66667,1.33333)$ and a global minimum at $(x, y)=(-0.86603,1.5)$. For this problem suppose that we start from point $\left(x_{0}, y_{0}\right)=(0.6,1.3)$.

1. Using solely $\Phi_{2}$, after 6 iterations we converge to $L^{*}=1.33333$ which is the above local maximum. 
2. Switching from $\Phi_{1}$ to $\Phi_{2}$ as in our algorithm, we converge after 13 iterations to $L^{*}=-1.29904$ which is the global minimum of the problem.

The algorithm presented in the preceding section has been implemented in standard C, and interfaced with AMPL. AMPL (Ref. 20) is a powerful mathematical modeling language that allows the optimization problems to be written in a simple algebraic notation, and also provides first and second order derivatives. The code was tested on a $2.4 \mathrm{GHz}$ workstation $2 \mathrm{~Gb}$ of memory. The test set consists of thirty-three minimax problems drawn from the CUTE set, available in AMPL format (Ref. 21).

The algorithm parameters were set as follows. In Step 1, the accuracy of the stopping criteria is $\epsilon_{0}=10^{-6}$. In Step 2.3, $\epsilon_{G}=10^{-4}$ and $\delta=10$. In Step 2.4, we set $\theta=0.9995, \beta=0.5, \rho=10^{-4}, m_{1}=1$ and $M_{1}=10$. In the barrier selection rule we set $\omega=6$.

The numerical results are summarized in Table 1, where we use the following abbreviations:

- Problem : The name of the CUTE set problem as described in Ref. 21.

- Itns : The total number of iterations required to find the optimal solution of the problem.

- $c_{0}$ : Initial value of the penalty parameter.

- $c_{*}$ : Final value of the penalty parameter.

- $k_{*}$ : The iteration after which the penalty parameter was unchanged.

- $c_{*}$ : the final value of the penalty parameter.

The majority starting points are the same as those in Ref. 21. For problems goffin, haifas and mifflin2 (0.0) was used instead. Additionally, for madsen we used $(0.3,1.0)$, for oet2 we used $(1.0)$, for polak1 $(1.0,0.05)$ and for polak6 $(-1.0,0.0,-1.0,0.0)$ were used as the starting point. Finally, for makela4 the first 10 variables were initialized to (1.0) and variables 11 to 20 were initialized to a value of $(-1.0)$.

The performance of the minimax algorithm in terms of the number of iterations is provided, along with the results obtained using LOQO, MINOS and IPOPT on the NEOS server 4.0. LOQO is an infeasible primal dual interior-point algorithm which uses a hybrid filter and merit function approach. MINOS 
uses a linear simplex method for linear problems, a reduced gradient method with quasi-Newton approximations for nonlinear objective functions, and sparse projected Lagrangian method for nonlinear constraints. IPOPT is an interior-point line search filter method that aims to find a local solution to general nonlinear programming problems. Our implementation does not permit timing experiments, or experiments on large scale problems since we use dense linear algebra operations. We hope that the addition of sparse large-scale linear algebra routines will allow a more elaborate comparison. In addition, in Table 1 we compare the performance of the algorithm when the single augmented Lagrangian merit function is used (column labelled $\Phi_{1}$ ) with the performance when both merit functions are used (column labelled $\left.\Phi_{1}, \Phi_{2}\right)$.

Table 1 shows, for the problems solved, that in many cases our algorithm compares favorably against MINOS, LOQO and IPOPT. For example, in problem haifam, where MINOS and LOQO failed, it took our algorithm 23 iterations and IPOPT was more than three times slower (74). Similarly, our algorithm solved problem polak3 in 20 iterations, a bit faster than LOQO (22), but MINOS failed and IPOPT was six times slower than our code (132). A similar result is obtained for problem polak6, where MINOS and IPOPT converged after 232 and 283 iterations, respectively, LOQO in 60 iterations, and our code in only 19 iterations (22 when switching merit functions). On the other hand, for problem oet2, MINOS failed, LOQO and our code were very slow (162 and 160 iterations, respectively) whereas IPOPT took only 19 iterations.

To demonstrate the switch between the two merit functions, the progress of the algorithm for problems chaconn1 and mifflin1 is shown in Table 2.

\section{Conclusions}

We have presented a primal dual-interior-point algorithm for constrained nonlinear, discrete minimax problems. In the inner iterations of the algorithm we employ a line search framework that uses two merit functions. One of the merit functions is the $l_{2}$ merit function. This merit function is initially used in the inner iteration to guide iterates towards feasibility. Once feasibility has been attained, a switch is made to the second merit function. The second merit function is the squared norm of the perturbed KKT residual. The latter is used for the rest of the inner iterations of the algorithm, i.e. until the perturbed KKT conditions have been satisfied. For the outer iterations 
of the algorithm an adaptive barrier parameter is used. Global convergence results have been presented. Numerical experimentation on small to medium scale problems shows that practical value of the proposed approach.

Acknowledgment. The authors would like to thank the two anonymous for their useful comments.

\section{References}

[1] Karmarkar, N., A New Polynomial-Time Algorithm for Linear Programming, Combinatorica, Vol.4, No.4, pp.373-395, 1984.

[2] El-Bakry, A. S., Tapia, R. A., Tsuchiya, T., and Zhang, Y., On the formulation and theory of the Newton interior-point method for nonlinear programming, Journal of Optimization Theory and Applications, Vol.89, No.3, pp.507-541, 1996.

[3] Kojima, M., Mizuno, S., And Yoshise, A., A primal-dual interior point method for linear programming, Progress in Mathematical Programming, Edited by N. Megiddo, New York, Springer-Verlag, pp. 29-47, 1989.

[4] Benson, H. Y., Shanno, D. F., and Vanderbei, R. J., Interiorpoint methods for nonconvex nonlinear programming: jamming and numerical testing, Mathematical Programming, Vol.99, No.1, Ser. A, pp.35-48, 2004.

[5] Du, D.-Z. and Pardalos, P. M., Minimax and Applications, Nonconvex Optimization and Its Applications, Springer, Dordrecht, Holland, 1995.

[6] Rustem, B. And Howe, M., Algorithms for worst-case design and applications to risk management, Princeton University Press, Princeton, NJ, 2002.

[7] Akrotirianakis, I. and Rustem, B., A Globally Convergent Interior Point Algorithm For Non-Linear Problems, Journal of Optimization Theory and Applications, Vol.125, No.3, pp.497-521, 2005. 
[8] Rustem, B., A constrained min-max algorithm for rival models of the same economic system, Mathematical Programming, Vol.53, No.3, Ser. A, pp.279-295, 1992.

[9] Medanić, J. And Andjelić, M., On a class of differential games without saddle-point solutions, Journal of Optimization Theory and Applications, Vol.8, pp.413-430, 1971.

[10] Medanić, J. And Andjelić, M., Minimax solution of the multipletarget problem, IEEE Transactions on Automatic Control, Vol.17, No.5, pp.597-604, 1972.

[11] Vanderbei, R. J. And Shanno, D. F., An interior point algorithm for nonconvex nonlinear programming, Computational Optimization and Applications, Vol.13, pp.231-252, 1999.

[12] Chamberlain, R. M., Powell, M. J. D., Lemarechal, C., And Pedersen, H. C., The Wathdog technique for Forcing Convergence in Algorithms for Constrained Optimization, Mathematical Programming Studies, Vol.16, pp.1-17, 1982.

[13] Gay, D. M., Overton, M. L., And Wright, M. H., A primaldual interior method for nonconvex nonlinear programming, Advances in nonlinear programming (Beijing, 1996), Edited by Y. Yuan, volume 14 of Appl. Optim. Kluwer Academic Publishers, Dordrecht, pp. 31-56, 1998.

[14] Rustem, B., Convergent Stepsizes for Constrained Optimization Algorithms, Journal of Optimization Theory and Applications, Vol.49, pp.135-160, 1986.

[15] Rustem, B., Equality and Inequality Constrained Optimization Algorithms with Convergent Stepsizes, Journal of Optimization Theory and Applications, Vol.76, No.3, pp.429-453, 1993.

[16] Rustem, B. And Nguyen, Q., An algorithm for the inequalityconstrained discrete min-max problem, SIAM Journal on Optimization, Vol.8, No.1, pp.265-283 (electronic), 1998.

[17] Yamashita, H., A globally convergent primal-dual interior point method for constrained optimization, Optimization Methods and Software, Vol.10, pp.443-469, 1998. 
[18] Yamashita, H. And Yabe, H., Superlinear and quadratic convergence of some primal-dual interior point methods for constrained optimization, Mathematical Programming, Vol.75, pp.377-397, 1996.

[19] Tzallas-Regas, G., Switching stepsize strategies for Nonlinear Programming, PhD thesis, University of London, Imperial College London, London, 2007.

[20] Fourer, R., Gay, D. M., and Kernighan, B. W., AMPL - A Modeling Language for Mathematical Programming, The Scientific Press, 1993.

[21] Benson, H., The Cute AMPL models, http://www.orfe.princeton. edu/ $\sim$ rvdb/ampl/nlmodels/cute, 1996. 


\section{List of Figures}

1 Graph for problem of Example 4.1 . . . . . . . . . . . . . 36

2 Graph for problem of Example 4.2 . . . . . . . . . . . 37 


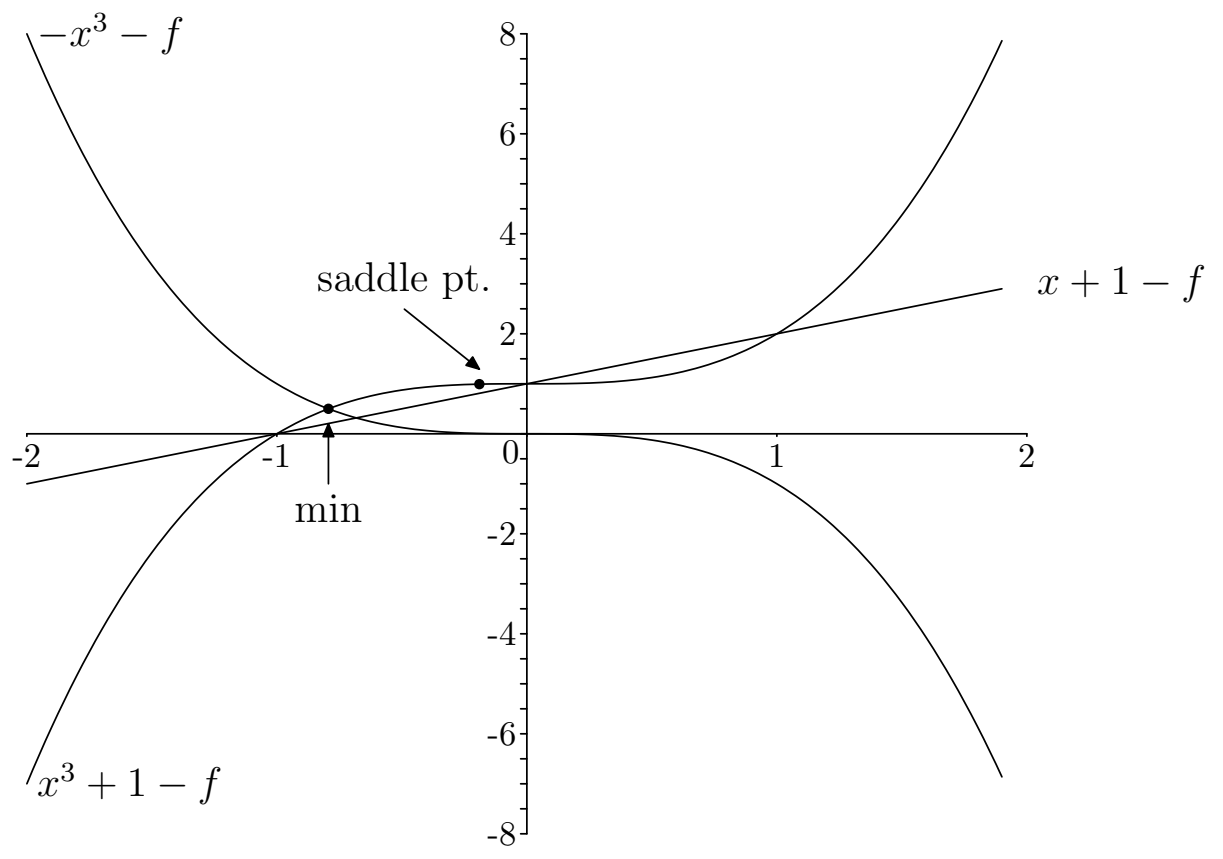

Figure 1: Graph for problem of Example 4.1 


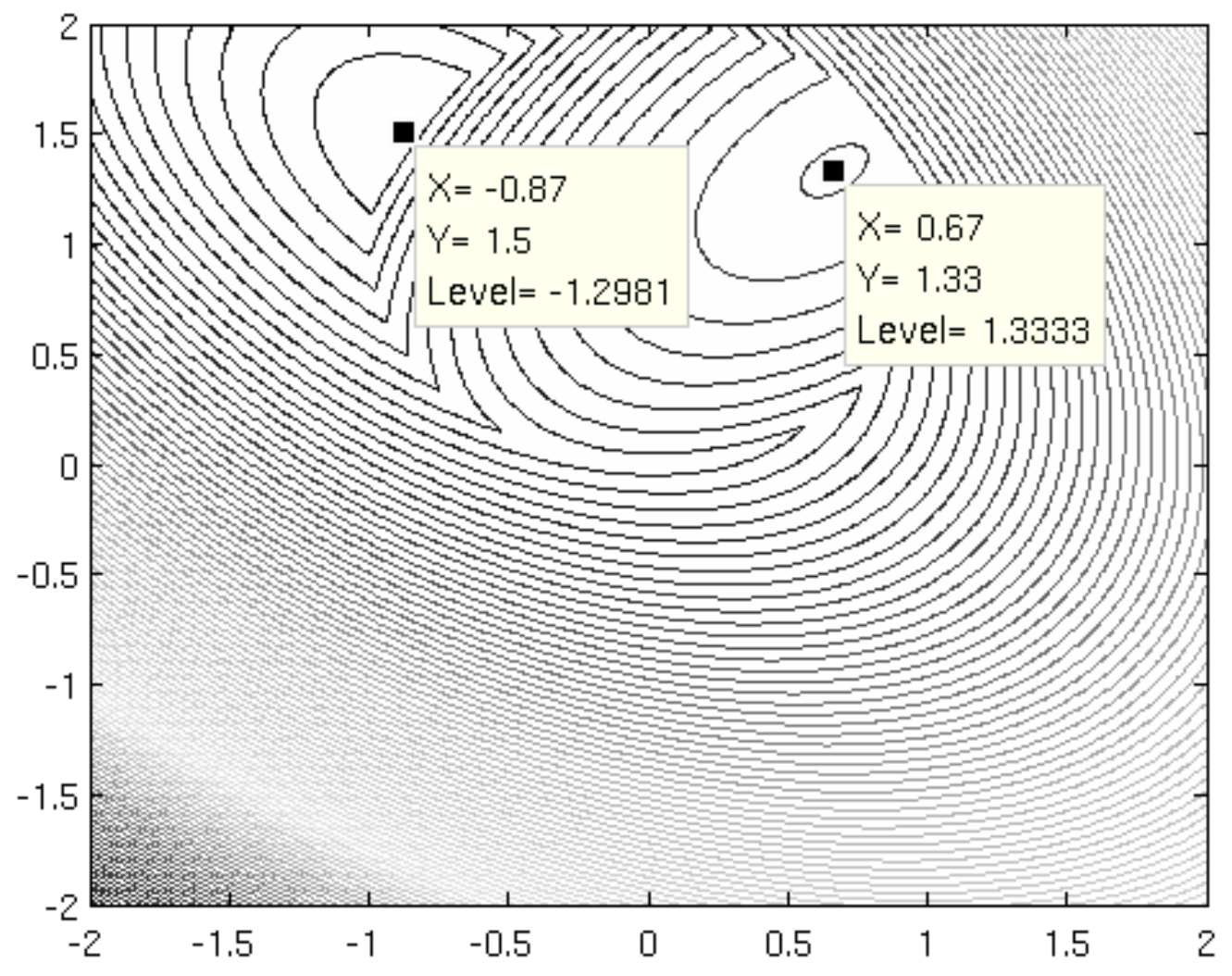

Figure 2: Graph for problem of Example 4.2 


\section{List of Tables}

1 Numerical results comparison . . . . . . . . . . . . . 39

2 Progress of the algorithm for chaconn1, mifflin. . . . . . . . 40 


\begin{tabular}{|c|c|c|c|c|c|c|c|c|c|c|}
\hline & MINOS & LOQO & IPOPT & \multicolumn{7}{|c|}{ Minimax algorithm } \\
\hline & \multirow[b]{2}{*}{ Itns } & \multirow[b]{2}{*}{ Itns } & \multirow[b]{2}{*}{ Itns } & \multicolumn{4}{|c|}{$\Phi_{1}$} & \multicolumn{3}{|c|}{$\Phi_{1}, \Phi_{2}$} \\
\hline & & & & $c_{0}$ & Itns & $c_{*}$ & $k_{*}$ & Itns & $c_{*}$ & $k_{*}$ \\
\hline cb2 & 11 & 8 & 7 & 0.1 & 8 & $6.39 \mathrm{e} 11$ & 8 & 8 & $1.71 \mathrm{e} 8$ & 6 \\
\hline cb3 & 2 & 9 & 6 & 0.1 & 9 & $4.53 \mathrm{e} 11$ & 9 & 9 & $6.94 \mathrm{e} 8$ & 7 \\
\hline chaconn1 & 24 & 9 & 7 & 0.1 & 9 & $3.08 \mathrm{e} 12$ & 9 & 9 & $3.7 \mathrm{e} 8$ & 7 \\
\hline chaconn 2 & 2 & 9 & 6 & 0.1 & 9 & $4.53 \mathrm{e} 11$ & 9 & 9 & $6.94 \mathrm{e} 8$ & 7 \\
\hline congigmz & 16 & 33 & 30 & 0.1 & 27 & $1.30 \mathrm{e} 12$ & 25 & 24 & $3.55 \mathrm{e} 8$ & 22 \\
\hline coshfun & 450 & 22 & 23 & 0.0 & 48 & $1.81 \mathrm{e} 11$ & 27 & 48 & $2.693 \mathrm{e} 6$ & 21 \\
\hline demymalo & 30 & 16 & 16 & 0.1 & 15 & $1.14 \mathrm{e} 12$ & 15 & 15 & $9.91 \mathrm{e} 7$ & 13 \\
\hline gigomez1 & 40 & 16 & 15 & 0.1 & 15 & $2.03 \mathrm{e} 10$ & 15 & 15 & $1.75 \mathrm{e} 8$ & 14 \\
\hline goffin & 1 & 11 & 6 & 0.0 & 3 & $4.88 \mathrm{e} 8$ & 3 & 3 & $4.88 \mathrm{e} 8$ & 3 \\
\hline haifam & $F^{a}$ & $\max ^{b}$ & 74 & 0.1 & 23 & $3.94 \mathrm{e} 7$ & 23 & 23 & $3.94 \mathrm{e} 7$ & 23 \\
\hline haifas & 59 & 12 & 8 & 0.1 & 10 & $1.03 \mathrm{e} 12$ & 10 & 10 & $4.55 \mathrm{e} 8$ & 8 \\
\hline kiwcresc & 17 & 14 & 20 & 10 & 11 & $9.45 \mathrm{e} 10$ & 11 & 11 & $8.51 \mathrm{e} 8$ & 10 \\
\hline madsen & 27 & 11 & 9 & 0 & 23 & $7.42 \mathrm{e} 8$ & 19 & 19 & $1.91 \mathrm{e} 6$ & 18 \\
\hline madsschj & 1083 & 22 & 22 & 0.0 & 29 & $3.81 \mathrm{e} 5$ & 29 & 29 & $3.81 \mathrm{e} 5$ & 29 \\
\hline makela1 & 26 & 14 & 18 & 1.0 & 13 & $1.69 \mathrm{e} 12$ & 13 & 13 & $1.19 \mathrm{e} 8$ & 11 \\
\hline makela2 & 21 & 12 & 8 & 0.1 & 11 & $2.63 \mathrm{e} 9$ & 11 & 11 & $7.5 \mathrm{e} 7$ & 10 \\
\hline makela3 & 22 & 11 & 6 & 0.1 & 10 & $1.65 \mathrm{e} 2$ & 8 & 10 & $1.65 \mathrm{e} 2$ & 8 \\
\hline makela4 & 1 & 11 & 6 & 0.1 & 4 & 0.1 & 1 & 4 & 0.1 & 1 \\
\hline mifflin1 & 24 & 9 & 7 & 0.1 & 9 & $2.81 \mathrm{e} 12$ & 9 & 9 & $2.52 \mathrm{e} 8$ & 7 \\
\hline mifflin2 & 11 & 10 & 8 & 1 & 10 & $4.34 \mathrm{e} 10$ & 10 & 12 & $6.2 \mathrm{e} 7$ & 8 \\
\hline minmaxrb & 24 & 10 & 10 & 1 & 15 & $1 \mathrm{e} 12$ & 15 & 17 & $2.94 \mathrm{e} 8$ & 15 \\
\hline oet1 & 159 & 14 & 39 & 0.0 & 17 & 730.57 & 1 & 17 & 730.57 & 1 \\
\hline oet2 & $F^{a}$ & 162 & 19 & 0.1 & 159 & $2.12 \mathrm{e} 10$ & 155 & 160 & $2.12 \mathrm{e} 10$ & 155 \\
\hline polak1 & $F^{a}$ & 11 & 6 & 0.1 & 7 & $2.04 \mathrm{e} 11$ & 7 & 7 & $5.3 \mathrm{e} 7$ & 5 \\
\hline polak2 & 136 & 17 & 19 & 0.1 & 9 & $2.44 \mathrm{e} 8$ & 9 & 9 & $2.44 \mathrm{e} 8$ & 9 \\
\hline polak3 & $F^{a}$ & 22 & 132 & 1 & 20 & $2.55 \mathrm{e} 12$ & 20 & 20 & 1 & 1 \\
\hline polak4 & 7 & 12 & 14 & 1 & 9 & $1.44 \mathrm{e} 8$ & 8 & 9 & $1.44 \mathrm{e} 8$ & 8 \\
\hline polak5 & 16 & 38 & 31 & 0.1 & 14 & $6.04 \mathrm{e} 8$ & 9 & 14 & $6.04 \mathrm{e} 8$ & 9 \\
\hline polak6 & 232 & 60 & 283 & 1 & 19 & $3.09 \mathrm{e} 8$ & 19 & 22 & $3.09 \mathrm{e} 8$ & 19 \\
\hline pt & 1 & 14 & 20 & 0.0 & 31 & $1.06 \mathrm{e} 12$ & 31 & 32 & $3.77 \mathrm{e} 8$ & 29 \\
\hline rosenmmx & 87 & 18 & 73 & 0.1 & 16 & $3.27 \mathrm{e} 8$ & 16 & 16 & $3.27 \mathrm{e} 8$ & 16 \\
\hline spiral & 62 & 24 & 17 & 1 & 23 & $1.41 \mathrm{e} 12$ & 22 & 23 & $8.32 \mathrm{e} 8$ & 17 \\
\hline womflet & 35 & 88 & 17 & 0.1 & 15 & $9 \mathrm{e} 11$ & 15 & 15 & $8.88 \mathrm{e} 8$ & 13 \\
\hline
\end{tabular}

${ }^{a}$ failure: superbasics limit too small.

${ }^{b}$ unable to solve within maximum number of iterations (default $\left.=500\right)$

Table 1: Numerical results comparison 


\begin{tabular}{|c|c|c|c|c|c|}
\hline Problem & Itns & Merit Function & $c$ & $f$ & $x_{k}$ \\
\hline \hline chaconn1 & & & & & \\
\hline & 1 & $\Phi_{1}$ & 10.1 & 3.94732 & $(1.00258,0.28172)$ \\
\hline & 2 & $\Phi_{1}$ & 10.1 & 2.02875 & $(1.17320,0.84020)$ \\
\hline & 3 & $\Phi_{1}$ & 10.1 & 2.00637 & $(1.12765,0.88404)$ \\
\hline & 4 & $\Phi_{1}$ & 10.1 & 1.95879 & $(1.13755,0.89775)$ \\
\hline & 5 & $\Phi_{1}$ & $2.6 \mathrm{e} 4$ & 1.95294 & $(1.13914,0.89915)$ \\
\hline & 6 & $\Phi_{1}$ & $3.3 \mathrm{e} 6$ & 1.95230 & $(1.13907,0.89950)$ \\
\hline & 7 & $\Phi_{1}$ & $3.7 \mathrm{e} 8$ & 1.95223 & $(1.13904,0.89955)$ \\
\hline & 8 & $\Phi_{2}$ & $3.7 \mathrm{e} 8$ & 1.95222 & $(1.13904,0.89956)$ \\
\hline & 9 & $\Phi_{2}$ & $3.7 \mathrm{e} 8$ & 1.95222 & $(1.13904,0.89956)$ \\
\hline \hline mifflin1 & & & & & \\
\hline & 1 & $\Phi_{1}$ & 45.08 & -0.04027 & $(0.99856,0.98040)$ \\
\hline & 2 & $\Phi_{1}$ & 45.08 & -0.654271 & $(1.13630,0.43687)$ \\
\hline & 3 & $\Phi_{1}$ & 45.08 & -0.87024 & $(1.11624,0.00233)$ \\
\hline & 4 & $\Phi_{1}$ & 45.08 & -0.982121 & $(1.0176,0.00003)$ \\
\hline & 5 & $\Phi_{1}$ & $1.9 \mathrm{e} 4$ & -0.99805 & $(1.00195,0)$ \\
\hline & 6 & $\Phi_{1}$ & $2.1 \mathrm{e} 6$ & -0.99980 & $(1.00020,0)$ \\
\hline & 7 & $\Phi_{1}$ & $2.5 \mathrm{e} 8$ & -0.99998 & $(1.00002,0)$ \\
\hline & 8 & $\Phi_{2}$ & $2.5 \mathrm{e} 8$ & -0.99999 & $(1,0)$ \\
\hline & 9 & $\Phi_{2}$ & $2.5 \mathrm{e} 8$ & -0.99999 & $(1,0)$ \\
\hline
\end{tabular}

Table 2: Progress of the algorithm for chaconn1, mifflin. 